\title{
Non-hyperoctahedral Categories of Two-Colored Partitions Part II: All Possible Parameter Values
}

\author{
Alexander Mang $^{1}$ (D) $\cdot$ Moritz Weber ${ }^{1}$ \\ Received: 7 July 2020 / Accepted: 25 March 2021 / Published online: 21 May 2021 \\ (c) The Author(s) 2021
}

\begin{abstract}
This article is part of a series with the aim of classifying all non-hyperoctahedral categories of two-colored partitions. Those constitute by some Tannaka-Krein type result the representation categories of a specific class of quantum groups. In Part I we introduced a class of parameters which gave rise to many new non-hyperoctahedral categories of partitions. In the present article we show that this class actually contains all possible parameter values of all nonhyperoctahedral categories of partitions. This is an important step towards the classification of all non-hyperoctahedral categories.
\end{abstract}

Keywords Quantum group · Unitary easy quantum group · Unitary group · Half-liberation · Tensor category · Two-colored partition · Partition of a set · Category of partitions · Brauer algebra

Mathematics Subject Classification 05A18 (Primary) · 20G42 (Secondary)

\section{Introduction}

In [8], Woronowicz provided a Tannaka duality for the (today so-called) compact matrix quantum groups he defined in [7] and which can be seen as a certain class of complex Hopf- $*$-algebras (compare also [9] for general compact quantum groups). More precisely, Woronowicz's theorem establishes a 2-equivalence between, on the one hand, the opposite of the $(2,1)$-category CMQG of compact matrix quantum groups and, on the other hand, the slice 2-category $\mathbf{g m} C^{*} \mathbf{C a t}_{\text {scfr }} / \mathrm{Hilb}_{\mathrm{f}}$ of small Cauchy-complete finite-dimensional rigid monoidal $C^{*}$-categories with a fixed a single-object generator (as 0 -cells and with unitary monoidal functors as 1-cells and unitary monoidal natural transformations as 2-cells) over the category of finite-dimensional complex Hilbert spaces.

Communicated by Stephen Lack.

$\bowtie \quad$ Alexander Mang

s9almang@stud.uni-saarland.de

Moritz Weber

weber@math.uni-sb.de

1 Fachbereich Mathematik, Saarland University, 66041 Saarbrücken, Germany 
Banica and Speicher showed in [1] how to construct 0-cells of the 2-category $\mathbf{g m C}^{*} \mathbf{C a t}_{\mathrm{scfr}} / \mathrm{Hilb}_{\mathrm{f}}$ from so-called categories of partitions, importantly utilizing combinatorics to produce heretofore scarce examples. Tarrago and the second author extended their construction in [6] to produce even more examples, now from categories of two-colored partitions. Moreover, they initiated a program to classify all such categories. The present article aims to further this effort.

Categories of two-colored partitions are explained in reference to a certain category $\mathcal{P}^{\circ \bullet}$ . The latter is defined to have as objects all words $c_{1} \ldots c_{k}$ over the alphabet $\{\circ, \bullet\}$. The morphism set from any word $c_{1} \ldots c_{k}$ to any word $d_{1} \ldots d_{\ell}$ consists of all set-theoretical partitions $p$ of $\left\{\boldsymbol{\bullet} 1, \ldots, k, \_1, \ldots, \ell\right\}$. Morphisms are composed by "vertical concatenation", which importantly involves the (associative operation of) forming the join of two set-theoretical partitions. The identity of $c_{1} \ldots c_{k}$ is the set containing exactly the sets $\{\because, i, i\}$ for all $i \in\{1, \ldots, k\}$. Morphisms are frequently depicted graphically. E.g., the identity morphism of $\circ \circ \bullet$ is addressed as $\$ ?:$.

Moreover, $\mathcal{P}^{\circ}$ is equipped with the strict monoidal structure given on objects by the ("horizontal") concatenation of words, $c_{1} \ldots c_{k} \otimes c_{1}^{\prime} \ldots c_{k^{\prime}}^{\prime}=c_{1} \ldots c_{k} c_{1}^{\prime} \ldots c_{k^{\prime}}^{\prime}$, and on morphisms by an operation in the same spirit. The monoidal unit object is the empty word $\varnothing$. The dagger functor acts by "reflection", i.e., exchanging " $i \leftrightarrow$. $i$. With respect to this monoidal structure, $\mathcal{P}^{\circ \bullet}$ is rigid. Moreover, it is generated as a rigid monoidal category by the single object given by the one-letter word $\circ$, whose dual object is $\bullet$. The (left) evaluation and co-evaluation morphisms of o correspond to $\mathcal{i}$ and $\square$, respectively. (That is enough to know because $\mathcal{P}^{\circ \bullet}$ can further be equipped with a symmetry, e.g., $\stackrel{9}{\bullet}$ from $\circ \bullet$ to $\bullet$ o.)

By definition, a category of two-colored partitions is now any wide (necessarily rigid) monoidal dagger-subcategory of $\mathcal{P}^{\circ}$ containing the evaluation and co-evaluation morphisms of $\circ$. Routinely, the symbol $\mathcal{P}^{\circ \bullet}$ is also used for the set of all morphisms of $\mathcal{P}^{\circ} \bullet$. And since categories of two-colored partitions are in particular supposed to be wide they are usually framed as subsets $\mathcal{C} \subseteq \mathcal{P}^{\circ \bullet}$ of this set $\mathcal{P}^{\circ}$, subject to corresponding closure conditions. (See [6] or [5] for an unabridged version of all those defini.)

Since the classification program for categories of two-colored partitions was begun, different subclasses have been indexed by various contributors (see [2-4,6]). The present article is the second part of a series aiming to determine and describe all so-called non-hyperoctahedral categories, i.e., all categories $\mathcal{C} \subseteq \mathcal{P}^{\circ \bullet}$ with $\hat{\imath} \otimes \hat{\bullet} \in \mathcal{C}$ or $\rfloor_{\bullet} \notin \mathcal{C}$.

In this regard the first article [5] and the present one pursue complementary approaches to detecting whether a given set of partitions is a non-hyperoctahedral category: Part I gave sufficient conditions for being a non-hyperoctahedral category, Part II now provides necessary ones.

Let us take a closer look at the findings of Part I, [5]. Every two-colored partition can be equipped with two natural structures on its set of points: a measure-like one, the color sum, and a metric-like one, the color distance. Both [5] and the present article study tuples of six properties of any given partition:

(1) the set of block sizes,

(2) the set of block color sums,

(3) the color sum of the set of all points,

(4) the set of color distances between subsequent legs of the same block with identical (normalized) colors,

(5) the set of color distances between subsequent legs of the same block with different (normalized) colors and

(6) the set of color distances between legs belonging to crossing blocks. 
By forming unions, one can aggregate these data over a given set of partitions. This information extracted from a set $\mathcal{S} \subseteq \mathcal{P}^{\circ \bullet}$ of partitions was called $Z(\mathcal{S})$ in [5].

There it was shown that one can give constraints on the above six properties which are preserved under category operations: A partially ordered set $(Q, \leq)$ of parameters was introduced to prove that the sets of the form

$$
\mathcal{R}_{Q}:=\left\{p \in \mathcal{P}^{\circ \bullet} \mid Z(\{p\}) \leq Q\right\} \text { for } Q \in Q
$$

form non-hyperoctahedral categories.

The current article now shows that these constraints encoded in $Z$ and $(Q, \leq)$ are natural in the following sense. (See also Section 2 for the definitions.)

Main Theorem [Theorem 9.1] Given any non-hyperoctahedral category $\mathcal{C} \subseteq \mathcal{P}^{\circ}$ of twocolored partitions, we have $Z(\mathcal{C}) \in Q$.

The importance of this result comes from its role in the overall program of the article series. On the one hand, it will be crucial to proving the main assertions of the ensuing articles. On the other hand, once those have been established, it will combine with them to show the final result of the entire series, roughly:

Main Theorem of the Series (Excerpt). Z restricts to a one-to-one correspondence between the set PCat ${ }_{\mathrm{NHO}}^{\circ}$ of non-hyperoctahedral categories of two-colored partitions and the parameter set $Q$.

The proof will go as follows: By Part I of the series, $\mathcal{R}_{Q} \subseteq P C a t_{\mathrm{NHO}}^{\circ}$ for every $Q \in Q$. Conversely, by the above Main Theorem of Part II, $Z(\mathcal{C}) \in Q$ for any $\mathcal{C} \in P C a t_{\text {NHO }}^{\circ}$. In the subsequent articles we will define a set $\mathcal{G}_{Z(\mathcal{C})} \subseteq \mathcal{P}^{\circ \bullet}$ and show

$$
\mathcal{G}_{Z(\mathcal{C})} \subseteq \mathcal{C} \subseteq\left\langle\mathcal{G}_{Z(\mathcal{C})}\right\rangle \text { and } \mathcal{G}_{Z\left(\mathcal{R}_{Z(\mathcal{C})}\right)} \subseteq \mathcal{R}_{Z(\mathcal{C})} \subseteq\left\langle\mathcal{G}_{Z\left(\mathcal{R}_{Z(\mathcal{C})}\right)}\right\rangle
$$

Proving $Z\left(\mathcal{R}_{Z(\mathcal{C})}\right)=Z(\mathcal{C})$ will then let us conclude $\mathcal{C}=\left\langle\mathcal{G}_{Z(\mathcal{C})}\right\rangle=\left\langle\mathcal{G}_{Z\left(\mathcal{R}_{Z(\mathcal{C})}\right)}\right\rangle=\mathcal{R}_{Z(\mathcal{C})}$.

\section{Reminder on Definitions from Part I}

For the convenience of the reader we briefly repeat those definitions from [5, Sections $3-$ 5] which are relevant to the current article. For definitions of partitions and categories of partitions see [5, Sections 3.1 and 4.2]. Throughout this article we will use the notations and definitions from [5, Sections 3-5].

Notation 2.1 For every set $S$ denote its power set by $\mathfrak{P}(S)$.

Definition 2.2 [5, Definition 5.2] The parameter domain $L$ is the sixfold Cartesian product of $\mathfrak{P}(\mathbb{Z})$.

Definition 2.3 [5, Definition 5.3] Using the notation from [5, Sections 3-5], we define the analyzer $Z: \mathfrak{P}\left(\mathcal{P}^{\circ}\right) \rightarrow L$ by

$$
Z:=(F, V, \Sigma, L, K, X)
$$

where, for all $\mathcal{S} \subseteq \mathcal{P}^{\circ}$,

(a) $F(\mathcal{S}):=\{|B| \mid p \in \mathcal{S}, B$ block of $p\}$ is the set of block sizes,

(b) $V(\mathcal{S}):=\left\{\sigma_{p}(B) \mid p \in \mathcal{S}, B\right.$ block of $\left.p\right\}$ is the set of block color sums, 
(c) $\Sigma(\mathcal{S}):=\{\Sigma(p) \mid p \in \mathcal{S}\}$ is the set of total color sums,

(d) $L(\mathcal{S}):=\left\{\delta_{p}\left(\alpha_{1}, \alpha_{2}\right) \mid p \in \mathcal{S}, B\right.$ block of $p, \alpha_{1}, \alpha_{2} \in B, \alpha_{1} \neq \alpha_{2}$,

$$
] \alpha_{1}, \alpha_{2}\left[{ }_{p} \cap B=\varnothing, \sigma_{p}\left(\left\{\alpha_{1}, \alpha_{2}\right\}\right) \neq 0\right\}
$$

is the set of color distances between any two subsequent legs of the same block having the same normalized color,

(e) $K(\mathcal{S}):=\left\{\delta_{p}\left(\alpha_{1}, \alpha_{2}\right) \mid p \in \mathcal{S}, B\right.$ block of $p, \alpha_{1}, \alpha_{2} \in B, \alpha_{1} \neq \alpha_{2}$,

$$
] \alpha_{1}, \alpha_{2}\left[p_{p} \cap B=\varnothing, \sigma_{p}\left(\left\{\alpha_{1}, \alpha_{2}\right\}\right)=0\right\}
$$

is the set of color distances between any two subsequent legs of the same block having different normalized colors and

(f) $X(\mathcal{S}):=\left\{\delta_{p}\left(\alpha_{1}, \alpha_{2}\right) \mid p \in \mathcal{S}, B_{1}, B_{2}\right.$ blocks of $p, B_{1}$ crosses $B_{2}$,

$$
\left.\alpha_{1} \in B_{1}, \alpha_{2} \in B_{2}\right\}
$$

is the set of color distances between any two legs belonging to two crossing blocks.

Notation $2.4 \quad$ (a) For all $x, y \in \mathbb{Z}$ and $A, B \subseteq \mathbb{Z}$ write

$$
x A+y B:=\{x a+y b \mid a \in A, b \in B\} .
$$

Moreover, put $x A-y B:=x A+(-y) B$. Per $A=\{1\}$ expressions like $x+y B$ are defined as well, and per $x=1$ so are such like $A+y B$.

(b) Let $\pm S:=S \cup(-S)$ for all sets $S \subseteq \mathbb{Z}$.

(c) For all $m \in \mathbb{Z}$ and $D \subseteq \mathbb{Z}$ define

$$
D_{m}:=(D \cup(m-D))+m \mathbb{Z} \text { and } D_{m}^{\prime}:=(D \cup(m-D) \cup\{0\})+m \mathbb{Z} .
$$

(d) Use the abbreviations $\llbracket 0 \rrbracket:=\varnothing$ and $\llbracket k \rrbracket:=\{1, \ldots, k\}$ for all $k \in \mathbb{N}$.

Definition 2.5 ([5, Definition 5.7]). Define the parameter range $Q$ as the subset of $L$ comprising all tuples $(f, v, s, l, k, x)$ listed below, where $u \in\{0\} \cup \mathbb{N}$, where $m \in \mathbb{N}$, where $\left.D \subseteq\{0\} \cup \llbracket \llbracket \frac{m}{2}\right\rfloor \rrbracket$, where $E \subseteq\{0\} \cup \mathbb{N}$ and where $N$ is a subsemigroup of $(\mathbb{N},+)$ :

\begin{tabular}{cccccc}
$f$ & $v$ & $s$ & $l$ & $k$ & $x$ \\
\hline$\{2\}$ & $\pm\{0,2\}$ & $2 u m \mathbb{Z}$ & $m \mathbb{Z}$ & $m \mathbb{Z}$ & $\mathbb{Z}$ \\
$\{2\}$ & $\pm\{0,2\}$ & $2 u m \mathbb{Z}$ & $m+2 m \mathbb{Z}$ & $2 m \mathbb{Z}$ & $\mathbb{Z}$ \\
$\{2\}$ & $\pm\{0,2\}$ & $2 u m \mathbb{Z}$ & $m+2 m \mathbb{Z}$ & $2 m \mathbb{Z}$ & $\mathbb{Z} \backslash m \mathbb{Z}$ \\
$\{2\}$ & $\{0\}$ & $\{0\}$ & $\varnothing$ & $m \mathbb{Z}$ & $\mathbb{Z}$ \\
$\{2\}$ & $\pm\{0,2\}$ & $\{0\}$ & $\{0\}$ & $\{0\}$ & $\mathbb{Z} \backslash N_{0}$ \\
$\{2\}$ & $\{0\}$ & $\{0\}$ & $\varnothing$ & $\{0\}$ & $\mathbb{Z} \backslash N_{0}$ \\
$\{2\}$ & $\{0\}$ & $\{0\}$ & $\varnothing$ & $\{0\}$ & $\mathbb{Z} \backslash N_{0}^{\prime}$ \\
$\{1,2\}$ & $\pm\{0,1,2\}$ & $u m \mathbb{Z}$ & $m \mathbb{Z}$ & $m \mathbb{Z}$ & $\mathbb{Z} \backslash D_{m}$ \\
$\{1,2\}$ & $\pm\{0,1,2\}$ & $2 u m \mathbb{Z}$ & $m+2 m \mathbb{Z}$ & $2 m \mathbb{Z}$ & $\mathbb{Z} \backslash D_{m}$ \\
$\{1,2\}$ & $\pm\{0,1\}$ & $u m \mathbb{Z}$ & $\varnothing$ & $m \mathbb{Z}$ & $\mathbb{Z} \backslash D_{m}$ \\
$\{1,2\}$ & $\pm\{0,1,2\}$ & $\{0\}$ & $\{0\}$ & $\{0\}$ & $\mathbb{Z} \backslash E_{0}$ \\
$\{1,2\}$ & $\pm\{0,1\}$ & $\{0\}$ & $\varnothing$ & $\{0\}$ & $\mathbb{Z} \backslash E_{0}$ \\
$\mathbb{N}$ & $\mathbb{Z}$ & $u m \mathbb{Z}$ & $m \mathbb{Z}$ & $m \mathbb{Z}$ & $\mathbb{Z} \backslash D_{m}$ \\
$\mathbb{N}$ & $\mathbb{Z}$ & $\{0\}$ & $\{0\}$ & $\{0\}$ & $\mathbb{Z} \backslash E_{0}$
\end{tabular}

The goal of this article, as sketched in the introduction, is to prove that $Z$ restricts to a map $P C a t_{\text {NHO }}^{\circ} \rightarrow Q$ (see Theorem 9.1). Evidently, $Q$ is not a Cartesian product; the six entries of the tuples cannot vary independently. Rather, only very special tuples of sets are allowed. Hence, if the claim $Z: P C a t_{\mathrm{NHO}}^{\circ \bullet} \rightarrow Q$ is to be true, then it is not enough to study the components of $Z$ individually. We must also investigate the relations between 
them. In consequence, the argument follows a winding path, taking components into and out of consideration underway as required or convenient.

\section{Tools: Equivalence and Projection}

We introduce an equivalence relation on pairs of partitions and consecutive sets therein by which to compare partitions locally (cf. [3, Definition 6.2]).

Definition 3.1 For all $i \in\{1,2\}$, let $P_{p_{i}}$ denote the set of all points of $p_{i} \in \mathcal{P}^{\circ \bullet}$ and let $S_{i} \subseteq P_{p_{i}}$ be consecutive. We call $\left(p_{1}, S_{1}\right)$ and $\left(p_{2}, S_{2}\right)$ equivalent if $S_{1}=S_{2}=\varnothing$ or if the following is true: There exist $n \in \mathbb{N}$ and for each $i \in\{1,2\}$ pairwise distinct points $\gamma_{i, 1}, \ldots, \gamma_{i, n}$ in $p_{i}$ such that $\left(\gamma_{i, 1}, \ldots, \gamma_{i, n}\right)$ is ordered in $p_{i}$ and $S_{i}=\left\{\gamma_{i, 1}, \ldots, \gamma_{i, n}\right\}$ and such that for all $j, j^{\prime} \in\{1, \ldots, n\}$ (possibly $j=j^{\prime}$ ) the following are true:

(1) The normalized colors of $\gamma_{1, j}$ in $p_{1}$ and $\gamma_{2, j}$ in $p_{2}$ agree.

(2) The points $\gamma_{1, j}$ and $\gamma_{1, j^{\prime}}$ both belong to a block $B_{1}$ of $p_{1}$ with $B_{1} \subseteq S_{1}$ if and only if $\gamma_{2, j}$ and $\gamma_{2, j^{\prime}}$ both belong to a block $B_{2}$ of $p_{2}$ with $B_{2} \subseteq S_{2}$.

(3) The points $\gamma_{1, j}$ and $\gamma_{1, j^{\prime}}$ both belong to a block $B_{1}$ of $p_{1}$ with $B_{1} \nsubseteq S_{1}$ if and only if $\gamma_{2, j}$ and $\gamma_{2, j^{\prime}}$ both belong to a block $B_{2}$ of $p_{2}$ with $B_{2} \nsubseteq S_{2}$.

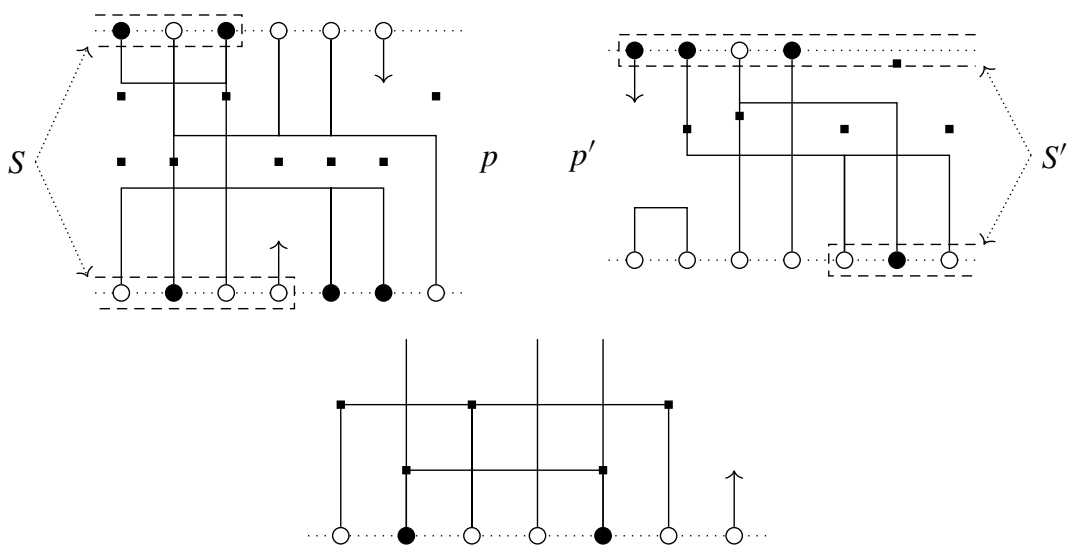

class of $(p, S) \cong\left(p^{\prime}, S^{\prime}\right)$

If $\left(p_{1}, S_{1}\right)$ and $\left(p_{2}, S_{2}\right)$ are equivalent, then $S_{1}$ and $S_{2}$ agree in size and normalized coloring up to a rotation $\varrho$ and the induced partitions $\left\{B_{1} \cap S_{1} \mid B_{1}\right.$ block of $\left.p_{1}\right\}$ of $S_{1}$ and $\left\{B_{2} \cap S_{2} \mid B_{2}\right.$ block of $\left.p_{2}\right\}$ of $S_{2}$ concur up to $\varrho$. However, this is only a necessary condition. Equivalence further requires that a block $B_{1} \cap S_{1}$ of the restriction of $p_{1}$ stems from a block $B_{1}$ of $p_{1}$ which has legs outside $S_{1}$ if and only if the corresponding statement $B_{2} \nsubseteq S_{2}$ is true for the block $B_{2}$ of $p_{2}$ which $B_{1}$ is mapped to under $\varrho$.

We define and construct special representatives of the classes of this equivalence relation. Recall that a partition $p \in \mathcal{P}^{\circ}$ is called projective if $p$ is self-adjoint, i.e., $p=p^{*}$, and idempotent, i.e., the pair $(p, p)$ is composable and $p p=p$.

Definition 3.2 For every consecutive set $S$ in $p \in \mathcal{P}^{\circ}$ we call the unique projective partition $q$ with lower row $M$ such that $(q, M)$ and $(p, S)$ are equivalent the projection $P(p, S)$ of $(p, S)$. 


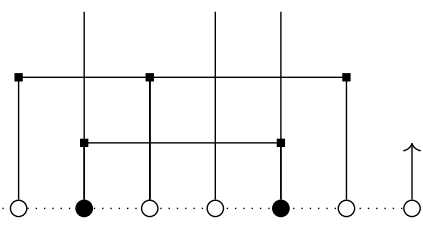

class of $(p, S) \cong(q, M)$

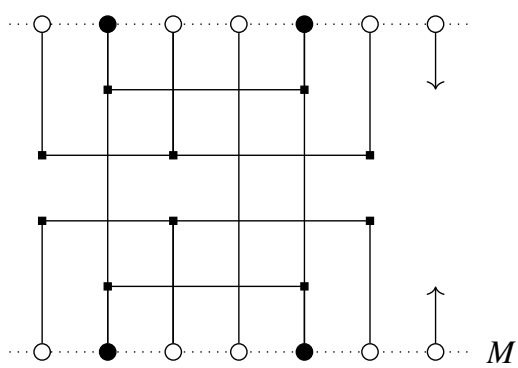

$P(p, S)=q$

In truth, of course, for any consecutive set $S$ in $p \in \mathcal{P}^{\circ \bullet}$ the projection $P(p, S)$ depends only on the equivalence class of $(p, S)$. The following lemma constitutes a generalization of [3, Lemma 6.4].

Lemma 3.3 $P(p, S) \in\langle p\rangle$ for any consecutive set $S$ in any $p \in \mathcal{P}^{\circ \bullet}$.

Proof As $S=\varnothing$ implies $P(p, S)=\varnothing \in\langle p\rangle$, let $S \neq \varnothing$. By rotation we can assume that $S$ is the lower row of $p$. Then $S$ has the same size and coloring in $p$ as in $q:=p p^{*}$. We show $q=P(p, S)$. By the nature of composition the blocks of $p$ which are contained in $S$ are blocks of $q$ as well. We only need to care about the other blocks of $q$. If we identify the upper row of $p$ and the lower row of $p^{*}$, the same partition $s$ is induced there by $p$ and $p^{*}$. Consequently, the meet of the two induced partitions is identical with $s$ as well. That means that every block $D$ of $s$ intersects exactly one block $B$ of $p$ and exactly one block of $p^{*}$, namely the mirror image of $B$. The block of $q$ resulting from $D$ therefore contains exactly the restriction of $B$ to the lower row and the mirror image of that set on the upper row. That means $q=P(p, S)$, which proves the claim.

\section{Step 1: Component $\boldsymbol{F}$ in Isolation}

We now take our first step towards proving the main result that the analyzer $Z$ from Definition 2.3 restricts to a map $P C a t_{\mathrm{NHO}}^{\circ} \rightarrow Q$ (see Theorem 9.1). Namely, we verify (see Proposition 4.3) that, for every non-hyperoctahedral category $\mathcal{C} \subseteq \mathcal{P}^{\circ}$, the set

$$
F(\mathcal{C}):=\{|B| \mid p \in \mathcal{C}, B \text { block of } p\}
$$

of block sizes appearing in $\mathcal{C}$ can only be one of the three sets of integers admissible as a first component for tuples in $Q$ by Definition 2.5.

Lemma 4.1 [6, Lemmata 1.3 (b), 2.1 (a) $]$ Let $\mathcal{C} \subseteq \mathcal{P}^{\circ \bullet}$ be a category.

(a) $\langle\hat{\imath} \otimes \hat{\bullet}\rangle=\langle\hat{\imath} \otimes \hat{0}\rangle=\left\langle\begin{array}{c}0 \\ \hat{0} \\ \hat{0}\end{array}\right\rangle=\left\langle\begin{array}{c}\hat{\imath} \\ \overrightarrow{0}\end{array}\right\rangle$.

(b) The following statements are equivalent:

(1) There exists in $\mathcal{C}$ a partition with a singleton block.

(2) $\hat{\imath} \otimes \hat{\imath} \in \mathcal{C}$.

(c) If $\hat{\imath} \otimes \uparrow \in \mathcal{C}$, then $\mathcal{C}$ is closed under disconnecting points from their blocks.

Proof (a) All transformations can be achieved by basic and cyclic rotations. 
(b) Projecting to a singleton block produces $\frac{2}{3}$ or $\frac{2}{3}$. Hence, Part (a) and Lemma 3.3 prove the claim.

(c) Rotate a given partition such that the leg to disconnect from its block is the only

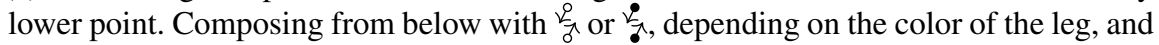
reversing the rotation achieves what is claimed. Hence, Part (a) concludes the proof.

Lemma $4.2[6$, Lemmata $1.3(\mathrm{~d}), 2.1$ (b) $]$ Let $\mathcal{C} \subseteq \mathcal{P}^{\circ \bullet}$ be a category.

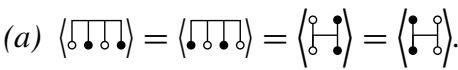

(b) The following statements are equivalent:

(1) There exists in $\mathcal{C}$ a partition with a block with at least three legs.

(2) $\square . . ! \in \mathcal{C}$.

(c) If $\square ! \in \mathcal{C}$, then $\mathcal{C}$ is closed under connecting the two points in any turn.

Proof (a) Once again, by basic and cyclic rotations we can transform the partitions into each other.

(b) Suppose $B$ is a block in $p \in \mathcal{C}$ with at least three legs, $\alpha, \beta \in B, \alpha \neq \beta$ and $] \alpha, \beta\left[{ }_{p} \cap B=\varnothing\right.$. Let $T$ be the set of the first lower and the first upper point of $P\left(p,[\alpha, \beta]_{p}\right)$. The partition $P\left(P\left(p,[\alpha, \beta]_{p}\right), T\right)$ is either $\because$ or $\mathfrak{H}_{0}$. Thus follows the claim by Part (a) and Lemma 3.3.

(c) Let $T$ be the turn in $p \in \mathcal{C}$ whose points we want to connect. By rotation we can assume that $T$ is the upper row of $p$. By composing $p$ from above with ${ }_{0}$ or $\mathfrak{H}_{0}$, depending on the sequence of colors in $T$, and reversing the initial rotation we achieve exactly what is claimed. So, Part (a) implies the assertion.

Recall the cases $\mathcal{O}, \mathcal{B}, \mathcal{S}$ from [5, Definition 4.1].

Proposition 4.3 Let $\mathcal{C} \subseteq \mathcal{P}^{\circ \bullet}$ be a non-hyperoctahedral category.

(a) The set $F(\mathcal{C})$ is given by $\{2\},\{1,2\}$ or $\mathbb{N}$.

(b) If $\mathcal{C}$ is case $\mathcal{O}$, then $F(\mathcal{C})=\{2\}$.

(c) If $\mathcal{C}$ is case $\mathcal{B}$, then $F(\mathcal{C})=\{1,2\}$.

(d) If $\mathcal{C}$ is case $\mathcal{S}$, then $F(\mathcal{C})=\mathbb{N}$.

Proof By definition of a category, $\square \in \mathcal{C}$ and thus $\{2\} \subseteq F(\mathcal{C})$.

(a) The first claim follows from the other three.

(b) Because $\hat{\imath} \otimes \hat{\imath} \notin \mathcal{C}$ and $\square . \emptyset \mathcal{C}$, Lemmata 4.1 (b) and 4.2 (b) show that every block in every partition of $\mathcal{C}$ has exactly two legs, i.e., $F(\mathcal{C})=\{2\}$.

(c) The assumption $\square ! \emptyset \notin \mathcal{C}$ implies by Lemma 4.2 (c) that no partition of $\mathcal{C}$ has blocks with more than two legs: $F(\mathcal{C}) \subseteq\{1,2\}$. Because $\hat{\imath} \otimes \uparrow \in \mathcal{C}$, it is clear that $\{1\} \subseteq F(\mathcal{C})$. Thus, $F(\mathcal{C})=\{1,2\}$ has been proven.

(d) It suffices to show $\mathbb{N} \subseteq F(\mathcal{C})$. Let $n \in \mathbb{N}$ be arbitrary. Then,

$$
p:=(\hat{0} \otimes \hat{\bullet})^{\otimes\left\lceil\frac{n}{2}\right\rceil} \in \mathcal{C} .
$$

Thanks to $]_{-} . \in \mathcal{C}$ we can, by Lemma 4.2 (c), connect the first $n$ points in $p$ to produce a partition in $\mathcal{C}$ containing a block with $n$ points, proving $\{n\} \subseteq F(\mathcal{C})$. 


\section{Step 2: Component $V$ and its Relation to $F$ and $L$}

The next objective is to narrow down the range of the component $V$ of $Z$ over $P C a t_{\mathrm{NHO}}^{\circ}$. Given a non-hyperoctahedral category $\mathcal{C} \subseteq \mathcal{P}^{\circ}$, we show that the set

$$
V(\mathcal{C}):=\left\{\sigma_{p}(B) \mid p \in \mathcal{C}, B \text { block of } p\right\}
$$

of block color sums occurring in $\mathcal{C}$ can only be one of the five sets allowed as second components for tuples of $Q$ by Definition 2.5. Beyond that, we can use Proposition 4.3 to show a result about the three parameters $V(\mathcal{C}), F(\mathcal{C})$ and

$$
\begin{gathered}
L(\mathcal{C}):=\left\{\delta_{p}\left(\alpha_{1}, \alpha_{2}\right) \mid p \in \mathcal{C}, B \text { block of } p, \alpha_{1}, \alpha_{2} \in B, \alpha_{1} \neq \alpha_{2},\right. \\
] \alpha_{1}, \alpha_{2}\left[{ }_{p} \cap B=\varnothing, \sigma_{p}\left(\left\{\alpha_{1}, \alpha_{2}\right\}\right) \neq 0\right\},
\end{gathered}
$$

the set of color distances between legs of the same block with identical normalized colors appearing in $\mathcal{C}$ : Viewed together as $(F, V, L)(\mathcal{C})$, they satisfy the conditions necessary for $Z(\mathcal{C})$ to be element of $Q$ by Definition 2.5.

Proposition 5.1 Let $\mathcal{C} \subseteq \mathcal{P}^{\circ}$ be a non-hyperoctahedral category.

(a) The set $V(\mathcal{C})$ is given by $\{0\}, \pm\{0,2\}, \pm\{0,1\}, \pm\{0,1,2\}$ or $\mathbb{Z}$.

(b) If $\mathcal{C}$ is case $\mathcal{O}$, then

$$
V(\mathcal{C})= \begin{cases} \pm\{0,2\} & \text { if } L(\mathcal{C}) \neq \varnothing \\ \{0\} & \text { otherwise }\end{cases}
$$

(c) If $\mathcal{C}$ is case $\mathcal{B}$, then

$$
V(\mathcal{C})= \begin{cases} \pm\{0,1,2\} & \text { if } L(\mathcal{C}) \neq \varnothing \\ \pm\{0,1\} & \text { otherwise. }\end{cases}
$$

(d) If $\mathcal{C}$ is case $\mathcal{S}$, then $L(\mathcal{C}) \neq \varnothing$ and $V(\mathcal{C})=\mathbb{Z}$.

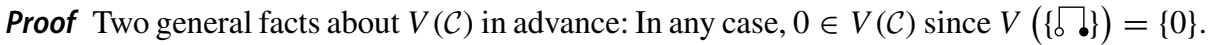
And [5, Lemma 6.4], using the fact that $p \in \mathcal{C}$ implies $\tilde{p} \in \mathcal{C}$, showed $V(\mathcal{C})=-V(\mathcal{C})$.

(a) Claim (a) follows from the other three.

(b) A pair block $B$ in $p \in \mathcal{C}$ satisfies $\sigma_{p}(B)=0$ if and only if that block has no two (necessarily subsequent) legs of the same normalized colors. Otherwise it has color sum -2 or 2 .

(c) And a singleton block always has color sums -1 or 1 . The rest follows from the proof of Part (b).

(d) If $\mathcal{C}$ is case $\mathcal{S}$, then $\square \in \in \mathcal{C}$ and $\uparrow \otimes \uparrow \in \mathcal{C}$. Hence, we can use $\uparrow \otimes \uparrow$ to disconnect

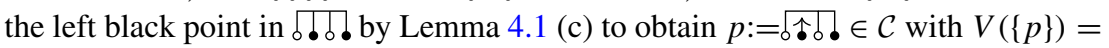
$\{-1,1\}$. Given any $n \in \mathbb{N}$, we use $\_.$. to connect in $p^{\otimes n} \in \mathcal{C}$ all the $n$ many threeleg blocks together (leaving the disconnected singletons alone) in accordance with Lemma 4.2 (c). That procedure results in the partition $q \in \mathcal{C}$ with $V(\{q\})=\{-1, n\}$. By $V(\mathcal{C})=-V(\mathcal{C})$ it then follows $V(\mathcal{C})=\mathbb{Z}$ as claimed.

\section{Step 3: Component $\Sigma$ in Isolation}

Easily, we can confirm that for all non-hyperoctahedral categories $\mathcal{C} \subseteq \mathcal{P}^{\circ}$ the set

$$
\Sigma(\mathcal{C}):=\{\Sigma(p) \mid p \in \mathcal{C}\}
$$


of all total color sums appearing in $\mathcal{C}$ is within the range of allowed third entries of tuples in $Q$ by Definition 2.5. The following proposition contains a generalization of [6, Lemma 2.6] and [6, Proposition 2.7].

Proposition 6.1 For every category $\mathcal{C} \subseteq \mathcal{P}^{\circ \bullet}$ the set $\Sigma(\mathcal{C})$ is a subgroup of $\mathbb{Z}$.

Proof [5, Lemma 6.5 (c)] implies $\Sigma(\mathcal{C})+\Sigma(\mathcal{C}) \subseteq \Sigma(\mathcal{C})$. And $-\Sigma(\mathcal{C}) \subseteq \Sigma(\mathcal{C})$ was shown in [5, Lemma 6.4]. As also $\Sigma(\square)=0$ and $\square \in \mathcal{C}$ by definition, the set $\Sigma(\mathcal{C})$ is indeed a subgroup of $\mathbb{Z}$.

\section{Step 4: General Relations between $\Sigma, L, K$ and $X$}

The goal remains proving that $Z$ (see Definition 2.3) maps the set $P C a t_{\mathrm{NHO}}^{\circ}$ of nonhyperoctahedral categories to $Q$ (see Definition 2.5). So far, we have tackled this problem, more or less, one component of $Z$ at a time. In that way, what we have managed to show is, mostly, that the values over $P C a t_{\mathrm{NHO}}^{\circ \bullet}$ of each of the three maps $F, V$ and $\Sigma$, viewed individually, are confined to the range of parameters allowed by $Q$ as corresponding entries of its elements. To complete this picture, we would also like to see that for any non-hyperoctahedral category $\mathcal{C} \subseteq \mathcal{P}^{\circ} \bullet$ the three sets $L(\mathcal{C})$,

$$
\begin{gathered}
K(\mathcal{C}):=\left\{\delta_{p}\left(\alpha_{1}, \alpha_{2}\right) \mid p \in \mathcal{C}, B \text { block of } p, \alpha_{1}, \alpha_{2} \in B, \alpha_{1} \neq \alpha_{2},\right. \\
] \alpha_{1}, \alpha_{2}\left[{ }_{p} \cap B=\varnothing, \sigma_{p}\left(\left\{\alpha_{1}, \alpha_{2}\right\}\right)=0\right\}
\end{gathered}
$$

and $X(\mathcal{C}):=\left\{\delta_{p}\left(\alpha_{1}, \alpha_{2}\right) \mid p \in \mathcal{C}, B_{1}, B_{2}\right.$ blocks of $p, B_{1}$ crosses $B_{2}$,

$$
\left.\alpha_{1} \in B_{1}, \alpha_{2} \in B_{2}\right\},
$$

too, can only be of the kinds allowed as fourth, fifth and sixth components of tuples in $Q$, respectively, by Definition 2.5. However, due to the strong interdependences between these three components of $Z$, it is not even possible to prove this basic claim about the ranges of the individual maps by studying them one at a time. Instead, now, the reasonable thing to do is to consider the tuple $(\Sigma, L, K, X)$ and make inferences about its range over $P C a t_{\mathrm{NHO}}^{\circ}$. That will give us (see Proposition 7.23) the claim about the individual ranges of $L, K$ and $X$ but also many more of the relations between them (and $\Sigma$ ), which we need to verify the main result.

\subsection{Abstract Arithmetic Lemma}

As a first step, it is best to study the relationship between the $\Sigma-, L-, K$ - and $X$-components of $Z$ in an abstract context, merely talking about arbitrary subsets of $\mathbb{Z}$ subject to certain axioms. Our goal for this subsection is to prove the Arithmetic Lemma (7.13): Assuming certain axioms (7.1), we may deduce a certain parameter range. We will show in Subsection 7.3 that for non-hyperoctahedral categories $\mathcal{C} \subseteq \mathcal{P}^{\circ \bullet}$ our sets $\Sigma(\mathcal{C}), L(\mathcal{C}), K(\mathcal{C})$ and $X(\mathcal{C})$ satisfy these axioms. Recall $\bar{\bullet}:=0$ and $\overline{0}:=\bullet$.

Axioms 7.1 Let $\sigma$ as well as $\kappa_{c_{1}, c_{2}}$ and $\xi_{c_{1}, c_{2}}$ for all $c_{1}, c_{2} \in\{0, \bullet\}$ be subsets of $\mathbb{Z}$. Throughout this subsection, make the following assumptions:

(i) $\sigma$ is a subgroup of $\mathbb{Z}$.

For all $\left(\omega_{c_{1}, c_{2}}\right)_{c_{1}, c_{2} \in\{0, \bullet\}} \in\left\{\left(\kappa_{c_{1}, c_{2}}\right)_{c_{1}, c_{2} \in\{\circ, \bullet\}},\left(\xi_{c_{1}, c_{2}}\right)_{c_{1}, c_{2} \in\{\circ, \bullet\}}\right\}$ and for all $c_{1}, c_{2} \in\{\circ, \bullet\}$ : 
(ii) $\omega_{c_{1}, c_{2}}+\sigma \subseteq \omega_{c_{1}, c_{2}}$.

(iii) $\omega_{c_{1}, c_{2}} \subseteq-\omega_{\overline{c_{2}}, \overline{c_{1}}}$.

(iv) $\omega_{c_{1}, c_{2}} \subseteq-\omega_{c_{2}, c_{1}}+\sigma$.

For all $c_{1}, c_{2}, c_{3} \in\{\circ, \bullet\}$ :

(v) $\xi_{c_{1}, c_{2}} \subseteq \xi_{c_{1}, \overline{c_{2}}} \cup\left(-\xi_{c_{2}, \overline{c_{1}}}+\sigma\right)$.

(vi) $0 \in \kappa_{\circ} \cap \cap \kappa_{\bullet}$.

(vii) $\kappa_{c_{1}, c_{2}}+\kappa_{\overline{c_{2}}, c_{3}} \subseteq \kappa_{c_{1}, c_{3}}$.

(viii) $\kappa_{c_{1}, c_{2}}+\xi_{\overline{c_{2}}, c_{3}} \subseteq \xi_{c_{1}, c_{3}}$.

Let us first study how much $\kappa_{c_{1}, c_{2}}$ and $\xi_{c_{1}, c_{2}}$ depend on $c_{1}, c_{2} \in\{\circ, \bullet\}$.

Lemma 7.2 For any $\left(\omega_{c_{1}, c_{2}}\right)_{c_{1}, c_{2} \in\{0, \bullet\}} \in\left\{\left(\kappa_{c_{1}, c_{2}}\right)_{c_{1}, c_{2} \in\{0, \bullet\}},\left(\xi_{c_{1}, c_{2}}\right)_{c_{1}, c_{2} \in\{0, \bullet\}}\right\}$ :

(a) $\omega_{\circ \circ}=\omega_{\bullet \bullet}$ and $\omega_{\circ \circ}=-\omega_{\circ \circ}=\omega_{\circ \circ}+\sigma$.

(b) $\omega_{\circ} \bullet \omega_{\bullet \circ}$ and $\omega_{\circ} \bullet=-\omega_{\circ} \bullet=\omega_{\circ}+\sigma$.

Proof Because $0 \in \sigma$ by Assumption (i), the Assumption (ii) actually means

$$
\omega_{c_{1}, c_{2}}=\omega_{c_{1}, c_{2}}+\sigma
$$

for all $c_{1}, c_{2} \in\{\circ, \bullet\}$. And with this new identity we can, for all $c_{1}, c_{2} \in\{\circ, \bullet\}$, refine Assumption (iv) to

$$
\omega_{c_{1}, c_{2}} \subseteq-\omega_{c_{2}, c_{1}}
$$

as $-\omega_{c_{2}, c_{1}}+\sigma=-\left(\omega_{c_{2}, c_{1}}-\sigma\right)=-\left(\omega_{c_{2}, c_{1}}+\sigma\right)=-\omega_{c_{2}, c_{1}}$ due to $\sigma=-\sigma$.

(a) Version (ii') of Assumption (ii) yields $\omega_{\circ \circ}=\omega_{\circ \circ}+\sigma$ as claimed. And Assumption (iv) in the form of (iv') proves

$$
\omega_{\circ \circ} \stackrel{(i v)}{\subseteq}-\omega_{\circ \circ} \stackrel{(i v)}{\subseteq} \omega_{\circ \circ} \text { and } \omega_{\bullet \bullet} \stackrel{(i v)}{\subseteq}-\omega_{\bullet \bullet} \stackrel{(i v)}{\subseteq} \omega_{\bullet \bullet},
$$

thus verifying $\omega_{\circ \circ}=-\omega_{\circ \circ}$ and $\omega_{\bullet \bullet}=-\omega_{\bullet \bullet}$. Now, if we apply Assumption (iii) to conclude

$$
\omega_{\circ \circ} \stackrel{(i i i)}{\subseteq}-\omega_{\bullet \bullet} \stackrel{(i i i)}{\subseteq} \omega_{\circ \circ},
$$

we can infer $\omega_{\circ \circ}=\omega_{\bullet \bullet}$. That proves the remainder of the claims about $\omega_{\circ \circ}$ and $\omega_{\bullet \bullet}$.

(b) Here also, Version (ii') of Assumption (ii) implies $\omega_{\circ} \bullet=\omega_{\circ} \bullet+\sigma$. Now, though, for $\omega_{\circ}$ and $\omega_{\bullet}$ the roles of Assumptions (iii) and (iv) reverse. First, we apply the former to conclude

$$
\omega_{\circ} \bullet \stackrel{(i i i)}{\subseteq}-\omega_{\circ} \stackrel{(\text { iiii) }}{\subseteq} \omega_{\circ \bullet} \text { and } \omega_{\bullet \circ} \stackrel{(i i i)}{\subseteq}-\omega_{\bullet \circ} \stackrel{(\text { iii })}{\subseteq} \omega_{\bullet \circ},
$$

which shows the claims $\omega_{\circ}=-\omega_{\bullet}$ and $\omega_{\bullet \circ}=-\omega_{\bullet \circ}$. Then, it is the refined version (iv') of Assumption (iv) that yields

$$
\omega_{\circ} \bullet \stackrel{(i v)}{\subseteq}-\omega_{\bullet \circ} \stackrel{(i v)}{\subseteq} \omega_{\circ},
$$

implying $\omega_{\circ \bullet}=\omega_{\bullet}$ and thus completing the proof.

In the case of $\left(\omega_{c_{1}, c_{2}}\right)_{c_{1}, c_{2} \in\{0, \bullet\}}=\left(\xi_{c_{1}, c_{2}}\right)_{c_{1}, c_{2} \in\{0, \bullet\}}$ of Lemma 7.2 we can go even further and combine the objects of Parts (a) and (b). 
Lemma $7.3 \xi_{\circ \circ}=\xi_{\circ}$.

Proof Since $\xi_{c_{2}, \overline{c_{1}}}=\xi_{c_{2}, \overline{c_{1}}}+\sigma$ for all $c_{1}, c_{2} \in\{\circ, \bullet\}$ by Version (ii') of Axiom (ii), our Assumption (v) actually spells

$$
\xi_{c_{1}, c_{2}} \subseteq \xi_{c_{1}, \overline{c_{2}}} \cup\left(-\xi_{c_{2}, \overline{c_{1}}}\right)
$$

for all $c_{1}, c_{2} \in\{\circ, \bullet\}$ as $\sigma=-\sigma$. Using this version of the assumption twice, we conclude

$$
\xi_{\circ \circ} \stackrel{(v)}{\subseteq} \xi_{\circ \bullet} \cup\left(-\xi_{\circ \bullet}\right)=\xi_{\circ \bullet} \stackrel{(v)}{\subseteq} \xi_{\circ \circ} \cup\left(-\xi_{\bullet \bullet}\right)=\xi_{\circ \circ},
$$

where we have used the results $\xi_{\circ}=-\xi_{\circ}$ and $\xi_{\circ \circ}=-\xi_{\bullet \bullet}$ of Lemma 7.2. It follows that indeed $\xi_{\circ \circ}=\xi_{\circ}$.

Definition 7.4 Write $\lambda:=\kappa_{\circ \circ}=\kappa_{\bullet \bullet}$ and $\kappa:=\kappa_{\circ \bullet}=\kappa_{\bullet \circ}$ and $\xi:=\xi_{\circ \circ}=\xi_{\bullet \bullet}=\xi_{\circ \bullet}=\xi_{\bullet \circ}$.

Our next step is to show that the pair $(\lambda, \kappa)$ is of a very simple form (Lemma 7.7).

Definition 7.5 Define the non-negative integers

$$
d:=\left\{\begin{array}{ll}
\min (\kappa \cap \mathbb{N}) & \text { if } \kappa \cap \mathbb{N} \neq \varnothing, \\
0 & \text { otherwise, }
\end{array} \quad \text { and } l:= \begin{cases}\min (\lambda \cap \mathbb{N}) & \text { if } \lambda \cap \mathbb{N} \neq \varnothing, \\
0 & \text { otherwise }\end{cases}\right.
$$

Lemma $7.6 \quad$ (a) $\kappa=d \mathbb{Z}$.

(b) If $\lambda \neq \varnothing$, then $l \in \lambda$ and $\lambda-l \supseteq \kappa$.

(c) $\lambda-l \subseteq \kappa$.

(d) If $\lambda \neq \varnothing$ and $d \neq 0$, then $l \leq d$.

(e) If $\lambda \neq \varnothing$ and $d \neq 0$, then $l \neq 0$.

(f) If $\lambda \neq \varnothing$, then $2 l \mathbb{Z} \subseteq d \mathbb{Z}$.

(g) If $\lambda \neq \varnothing$, then $d=l$ or $d=2 l$.

Proof (a) Of course, $0 \in \kappa$ by Assumption (vi). And $-\kappa=\kappa$ was established in Lemma 7.2 (b). And with the choices $c_{1}=0, c_{2}=c_{3}=\bullet$, Assumption (vii) implies that

$$
\kappa+\kappa=\kappa_{\circ} \bullet+\kappa_{\circ} \stackrel{(v i i)}{\subseteq} \kappa_{\circ}=\kappa .
$$

Hence, $\kappa$ is indeed a subgroup of $\mathbb{Z}$. The definition of $d$ makes $d$ a generator of $\kappa$, implying $\kappa=d \mathbb{Z}$.

(b) As $\lambda=-\lambda$ by Lemma 7.2 (a), assuming $\lambda \neq \varnothing$ ensures $\lambda \cap(\{0\} \cup \mathbb{N}) \neq \varnothing$. Hence, under this assumption, $l \in \lambda$ by definition of $l$. If we choose $c_{1}=c_{3}=\circ$ and $c_{2}=\bullet$ in Assumption (vii), it follows that

$$
\kappa+\lambda=\kappa_{\circ} \bullet+\kappa_{\circ \circ} \stackrel{(v i i)}{\subseteq} \kappa_{\circ \circ}=\lambda .
$$

Since $l \in \lambda$, we can specialize the $\lambda$ on the left hand side of that inclusion to $l$ and then subtract $l$ on both sides. We obtain $\kappa \subseteq \lambda-l$.

(c) If $\lambda=\varnothing$, there is nothing to prove. Hence, let $\lambda \neq \varnothing$, implying $l \in \lambda$ by Part (b). Using Assumption (vii) once more, this time with the choices $c_{1}=c_{2}=\circ$ and $c_{3}=\bullet$, yields

$$
\lambda-\lambda=\lambda+\lambda=\kappa_{\circ \circ}+\kappa_{\bullet \bullet} \stackrel{(v i i)}{\subseteq} \kappa_{\circ} \bullet=\kappa,
$$

where we have used $\lambda=-\lambda$ (Lemma 7.2 (a)) in the first step. Specializing on the left hand side the second instance of $\lambda$ to $l$ yields $\lambda-l \subseteq \kappa$. 
(d) Actually, we show the contraposition. Hence, suppose $\lambda \neq \varnothing$ and $l>d$. Since $\lambda=l+d \mathbb{Z}$ by Parts (a)-(c), it then follows that $l-d \in \lambda \cap \mathbb{N}$. The definition of $l$ consequently requires $l \leq l-d$, i.e. $d \leq 0$. As $d \geq 0$ by definition, $d=0$ is the only possibility.

(e) We prove the contraposition indirectly. As $\lambda=l+d \mathbb{Z}$ by Parts (a)-(c), supposing $l=0$ entails $\lambda=d \mathbb{Z}$. Thus, if $d \neq 0$ were true, then $\varnothing \neq d \mathbb{Z} \cap \mathbb{N}=\lambda \cap \mathbb{N}$ would yield the contradiction $0<\min (\lambda \cap \mathbb{N})=l=0$ by definition of $l$.

(f) In the proof of Part (c) we saw $\lambda+\lambda \subseteq \kappa$. Specializing therein both instances of $\lambda$ on the left hand side to $l$ (which we can do due to $\lambda \neq \varnothing$ by Part (b)) yields $2 l \in \kappa=d \mathbb{Z}$. It follows $2 l \mathbb{Z} \subseteq d \mathbb{Z}$ as asserted.

(g) From $2 l \mathbb{Z} \subseteq d \mathbb{Z}$, as shown in Part (f), it is immediate that, if $d=0$, then $l=0=d$ as claimed. If $d \neq 0$, we know, firstly, $l \leq d$ by Part (d), secondly, $l \neq 0$ by Part (e) and, thirdly, $2 l \mathbb{Z} \subseteq d \mathbb{Z}$ by Part (f). That is only possible if $d=l$ or $d=2 l$ : Indeed, if $c \in \mathbb{Z}$ is such that $2 l=c d$, then $l>0$ and $d \geq 0$ ensure $c>0$. Moreover, $l \leq d$ implies $2 l \leq 2 d$, i.e., $c d \leq 2 d$. We infer $c \leq 2$ by $d>0$. Hence, $c \in\{1,2\}$ by $c>0$.

Lemma 7.7 (a) If $\lambda=\varnothing$, then $(\lambda, \kappa)=(\varnothing, d \mathbb{Z})$.

(b) If $\lambda \neq \varnothing$, then $(\lambda, \kappa)$ is equal to $(l+2 l \mathbb{Z}, 2 l \mathbb{Z})$ or $(l \mathbb{Z}, l \mathbb{Z})$.

Proof In Lemma 7.6 we established that $\kappa=d \mathbb{Z}$ (Part (a)) and that $\lambda=\varnothing$ or $\lambda=l+d \mathbb{Z}$ (Parts (b) and (c)), where $d=l$ or $d=2 l$ (Part (g)). In other words, we have proven that $(\lambda, \kappa)$ is of the asserted form.

We can immediately relate $\sigma$ to $\kappa$.

Definition 7.8 Define

$$
k:= \begin{cases}\min (\sigma \cap \mathbb{N}) & \text { if } \sigma \cap \mathbb{N} \neq \varnothing, \\ 0 & \text { otherwise. }\end{cases}
$$

Lemma $7.9 \sigma=k \mathbb{Z} \subseteq d \mathbb{Z}=\kappa$.

Proof Because $\sigma$ is a subgroup of $\mathbb{Z}$, the definition of $k$ implies $\sigma=k \mathbb{Z}$. Moreover, we know $\kappa=\kappa+\sigma$ by Lemma 7.2 (b). Hence Assumption (vi), namely $0 \in \kappa$, implies $k \mathbb{Z}=\sigma \subseteq \kappa+\sigma \subseteq \kappa=d \mathbb{Z}$.

Let us now turn to the description of $\xi$.

Lemma 7.10 (a) $\xi=\xi+d \mathbb{Z}$.

(b) If $\lambda \neq \varnothing$, then $\xi=\xi+l \mathbb{Z}$.

Proof (a) Picking $c_{1}=\circ, c_{2}=c_{3}=\bullet$, Assumption (viii) implies the inclusion

$$
\kappa+\xi=\kappa_{\circ} \bullet+\xi_{\circ} \stackrel{(v i i i)}{\subseteq} \xi_{\circ \bullet}=\xi .
$$

As the reverse inclusion is trivially true by $0 \in \kappa$ (Assumption (vi)), we have thus verified our claim $\xi=\xi+d \mathbb{Z}$ by Lemma 7.6 (a).

(b) Assumption (viii), applied a second time, now with $c_{1}=c_{2}=c_{3}=\circ$, allows us to conclude

$$
\lambda+\xi=\kappa_{\circ \circ}+\xi_{\bullet \circ} \stackrel{(\text { viii })}{\subseteq} \xi_{\circ \circ}=\xi
$$


If $\lambda \neq \varnothing$, then $l \in \lambda$ by Lemma 7.6 (b). Hence, the above inclusion shows in particular $\xi+l \subseteq \xi$. Using this, induction proves $\xi+l \mathbb{N} \subseteq \xi$. Lemma 7.6 (g) established that $d=l$ or $d=2 l$. Either way, $\xi=\xi+d \mathbb{Z}$, as seen in Part (a), then ensures $\xi-2 l \subseteq \xi$. Combining this conclusion with $\xi+l \subseteq \xi$ lets us infer $\xi-l=(\xi+l)-2 l \subseteq \xi$. Again, it follows $\xi-l \mathbb{N} \subseteq \xi$ by induction. Hence, altogether we have shown $\xi+l \mathbb{Z}=$ $(\xi-l \mathbb{N}) \cup \xi \cup(\xi+l \mathbb{N}) \subseteq \xi$. Of course, the converse inclusion is true as well because $0 \in \mathbb{Z}$, proving $\xi=\xi+l \mathbb{Z}$ as claimed.

In order to obtain a refined understanding of $\xi$ we need the following preparatory lemma.

Lemma 7.11 Let $\chi \subseteq \mathbb{Z}$ and $m \in \mathbb{N}$ satisfy $\chi=-\chi=\chi+m \mathbb{Z}$.

(a) $\chi=(\chi \cap(\{0\} \cup \llbracket m-1 \rrbracket))_{m}$.

(b) $\chi \cap \llbracket m-1 \rrbracket=m-(\chi \cap \llbracket m-1 \rrbracket)$.

(c) $\left.\chi=\left(\chi \cap\left(\{0\} \cup \llbracket \llbracket \frac{m}{2}\right\rfloor \rrbracket\right)\right)_{m}$.

(d) $\chi=\mathbb{Z} \backslash D_{m}$ for $D=\left(\{0\} \cup \llbracket\left\lfloor\frac{m}{2}\right\rfloor \rrbracket\right) \backslash \chi$.

Proof The mapping $S \mapsto S_{m}:=(S \cup(m-S))+m \mathbb{Z}$ of subsets $S \subseteq \mathbb{Z}$ is a closure operator with respect to $\subseteq$, i.e., for all $S, T \subseteq \mathbb{Z}$ with $S \subseteq T$ we have $S \subseteq S_{m}$ and $S_{m} \subseteq T_{m}$ and $\left(S_{m}\right)_{m}=S_{m}$. In particular $S=S_{m}$ if and only if $S=-S=S+m \mathbb{Z}$.

(a) The assumption $\chi=-\chi=\chi+m \mathbb{Z}$ implies $\chi=\chi_{m}$. Hence, $\chi=\chi_{m} \supseteq(\chi \cap$ $(\{0\} \cup \llbracket m-1 \rrbracket))_{m}$ is clear by monotonicity of $S \mapsto S_{m}$. We show the converse: If $x \in \chi$, we find $x^{\prime} \in\{0\} \cup \llbracket m-1 \rrbracket$ such that $x^{\prime}-x \in m \mathbb{Z}$. Consequently, $x^{\prime} \in x+m \mathbb{Z} \subseteq \chi+m \mathbb{Z} \subseteq \chi$ by assumption. We conclude $x \in x^{\prime}+m \mathbb{Z} \subseteq$ $(\chi \cap(\{0\} \cup \llbracket m-1 \rrbracket))+m \mathbb{Z} \subseteq(\chi \cap(\{0\} \cup \llbracket m-1 \rrbracket))_{m}$, which is what we needed to show.

(b) We further deduce from $\chi=-\chi=\chi+m \mathbb{Z}$ that $m-\chi \subseteq \chi$. Naturally, $m-(\chi \cap \llbracket m-1 \rrbracket) \subseteq m-\llbracket m-1 \rrbracket=\llbracket m-1 \rrbracket$. Combining this with $m-(\chi \cap \llbracket m-1 \rrbracket) \subseteq m-\chi \subseteq \chi$ yields $m-(\chi \cap \llbracket m-1 \rrbracket) \subseteq \chi \cap \llbracket m-1 \rrbracket$. We conclude $\chi \cap \llbracket m-1 \rrbracket=m-(m-(\chi \cap \llbracket m-1 \rrbracket)) \subseteq m-(\chi \cap \llbracket m-1 \rrbracket)$, which proves one inclusion.

Now, the converse. From $\chi=-\chi=\chi+m \mathbb{Z}$ we can infer $m-\chi=-(m-\chi)=$ $(m-\chi)+m \mathbb{Z}$. In consequence we can apply the inclusion we just proved to the set $m-\chi$ in the role of $\chi$. Since $m-\llbracket m-1 \rrbracket=\llbracket m-1 \rrbracket$, the resulting inclusion $(m-\chi) \cap \llbracket m-1 \rrbracket \subseteq m-((m-\chi) \cap \llbracket m-1 \rrbracket)$ actually spells $m-(\chi \cap \llbracket m-1 \rrbracket) \subseteq \chi \cap \llbracket m-1 \rrbracket$. That is just what we had to show.

(c) Due to the monotonicity and idempotency of the mapping $S \mapsto S_{m}$, it suffices by Part (a) to prove $\chi \cap(\{0\} \cup \llbracket m-1 \rrbracket) \subseteq\left(\chi \cap\left(\{0\} \cup \llbracket\left\lfloor\frac{m}{2}\right\rfloor \rrbracket\right)\right)_{m}$. Let $x \in \chi \cap$ $(\{0\} \cup \llbracket m-1 \rrbracket)$ be arbitrary. If $x \leq\left\lfloor\frac{m}{2}\right\rfloor$, then, naturally, $x \in \chi \cup\left(\{0\} \cup \llbracket\left\lfloor\frac{m}{2}\right\rfloor \rrbracket\right) \subseteq$ $\left(\chi \cap\left(\{0\} \cup \llbracket\left\lfloor\frac{m}{2}\right\rfloor \rrbracket\right)\right)_{m}$. Hence, we can assume $x>\left\lfloor\frac{m}{2}\right\rfloor$. By Part (b) we know $m-x \in \chi$. By assumption, $m-x<m-\left\lfloor\frac{m}{2}\right\rfloor$. If $m$ is even, then this inequality says $m-x<m-\frac{m}{2}=\frac{m}{2}=\left\lfloor\frac{m}{2}\right\rfloor$. Should $m$ be odd instead, it means $m-$ $x<m-\frac{m-1}{2}=\frac{m+1}{2}$, which implies $m-x \leq \frac{m+1}{2}-1=\frac{m-1}{2}=\left\lfloor\frac{m}{2}\right\rfloor$. Thus, $m-x \leq\left\lfloor\frac{m}{2}\right\rfloor$ in all cases. Hence we have shown $m-x \in \chi \cap\left(\{0\} \cup \llbracket\left\lfloor\frac{m}{2}\right\rfloor \rrbracket\right)$. It follows $x=m-(m-x) \in m-\left(\chi \cap\left(\{0\} \cup \llbracket\left\lfloor\frac{m}{2}\right\rfloor \rrbracket\right)\right) \subseteq\left(\chi\left(\{0\} \cup \llbracket\left[\frac{m}{2}\right\rfloor \rrbracket\right)\right)_{m}$. That is what we needed to see.

(d) The assumption $\chi=-\chi=\chi+m \mathbb{Z}$ implies $\mathbb{Z} \backslash \chi=-(\mathbb{Z}$ a ckslash $\chi)=(\mathbb{Z} \backslash \chi)+$ $m \mathbb{Z}$. Hence, we can apply Part (c) to the set $\mathbb{Z} \backslash \chi$ in the role of $\chi$ and obtain $\mathbb{Z} \backslash \chi=$ $\left((\mathbb{Z} \backslash \chi) \cap\left(\{0\} \cup \mathbb{[}\left\lfloor\frac{m}{2}\right\rfloor \rrbracket\right)\right)_{m}$. Since $(\mathbb{Z} \backslash \chi) \cap\left(\{0\} \cup \mathbb{[}\left\lfloor\frac{m}{2}\right\rfloor \rrbracket\right)=\left(\{0\} \cup \llbracket\left\lfloor\frac{m}{2}\right\rfloor \rrbracket\right) \backslash \chi=D$ we have shown $\mathbb{Z} \backslash \chi=D_{m}$. It follows $\chi=\mathbb{Z} \backslash D_{m}$ as claimed. 
Lemma 7.12 (a) If $d=0$, then $\xi=\mathbb{Z} \backslash E_{0}$ for $E=(\{0\} \cup \mathbb{N}) \backslash \xi$.

(b) If $d \geq 1$ and $\lambda \neq \varnothing$, then $\xi=\mathbb{Z} \backslash D_{l}$ for $D=\left(\{0\} \cup \llbracket\left\lfloor\frac{l}{2}\right\rfloor \rrbracket\right) \backslash \xi$.

(c) If $d \geq 1$ and $\lambda=\varnothing$, then $\xi=\mathbb{Z} \backslash D_{d}$ for $D=\left(\{0\} \cup \llbracket\left\lfloor\frac{d}{2}\right\rfloor \rrbracket\right) \backslash \xi$.

Proof (a) The defining equations $E=(\{0\} \cup \mathbb{N}) \backslash \xi$ and $E_{0}=E \cup(-E)$ imply $E_{0}=$ $((\{0\} \cup \mathbb{N}) \backslash \xi) \cup((-(\{0\} \cup \mathbb{N})) \backslash(-\xi))$. Hence, $\xi=-\xi$ (by Lemma 7.2) shows $E_{0}=\mathbb{Z} \backslash \xi$ and thus the claim $\xi=\mathbb{Z} \backslash E_{0}$.

(b) Because $\lambda \neq \varnothing$, Lemma $7.6(\mathrm{~g})$ guarantees $d=l$ or $d=2 l$. Hence, the assumption $d \geq 1$ implies $l \geq 1$. Moreover, Lemma 7.10 (b) assures us that $\xi=\xi+l \mathbb{Z}$. And, we already know $\xi=-\xi$ by Lemma 7.2. Hence, Lemma 7.11 (d) yields the claim.

(c) Still, $\xi=-\xi$, of course. And $\xi=\xi+d \mathbb{Z}$ by Lemma 7.10 (a) as $d \geq 1$. Thus, once more, Lemma 7.11 (d) proves the claim.

In conclusion we have shown the following auxiliary result.

Lemma 7.13 (Arithmetic Lemma). If the nine sets of integers $\sigma$ and $\kappa_{c_{1}, c_{2}}, \xi_{c_{1}, c_{2}}$ for $c_{1}, c_{2} \in$ $\{\circ, \bullet\}$ satisfy Axioms 7.1, then

$$
\kappa_{\circ \circ}=\kappa_{\bullet \bullet}=: \lambda, \quad \kappa_{\circ \bullet}=\kappa_{\bullet \circ}=: \kappa \text { and } \xi_{\circ \circ}=\xi_{\bullet \bullet}=\xi_{\circ \bullet}=\xi_{\bullet \circ}=: \xi
$$

and there exist $u \in\{0\} \cup \mathbb{N}, m \in \mathbb{N}, D \subseteq\{0\} \cup \llbracket\left\lfloor\frac{m}{2}\right\rfloor \rrbracket$ and $E \subseteq\{0\} \cup \mathbb{N}$ such that the tuple $(\sigma, \lambda, \kappa, \xi)$ is given by one of the following:

\begin{tabular}{cccc}
$\sigma$ & $\lambda$ & $\kappa$ & $\xi$ \\
\hline$u m \mathbb{Z}$ & $m \mathbb{Z}$ & $m \mathbb{Z}$ & $\mathbb{Z} \backslash D_{m}$ \\
$2 u m \mathbb{Z}$ & $m+2 m \mathbb{Z}$ & $2 m \mathbb{Z}$ & $\mathbb{Z} \backslash D_{m}$ \\
$u m \mathbb{Z}$ & $\varnothing$ & $m \mathbb{Z}$ & $\mathbb{Z} \backslash D_{m}$ \\
$\{0\}$ & $\{0\}$ & $\{0\}$ & $\mathbb{Z} \backslash E_{0}$ \\
$\{0\}$ & $\varnothing$ & $\{0\}$ & $\mathbb{Z} \backslash E_{0}$
\end{tabular}

Proof That $\lambda, \kappa$ and $\xi$ are well-defined was shown in Lemmata 7.2 and 7.3. Hence, we can let $k, d$ and $l$ be as in Definitions 7.8 and 7.5. We distinguish five cases in total.

Case 1: First, suppose that $\lambda=\varnothing$. Then, $\kappa=d \mathbb{Z}$. By Lemma 7.7 (a). There are now two possibilities depending on the value of $d \in\{0\} \cup \mathbb{N}$.

Case 1.1: If $d=0$, which is to say $\kappa=\{0\}$, then Lemma 7.12 (a) yields $\xi=\mathbb{Z} \backslash E_{0}$ for $E:=(\{0\} \cup \mathbb{N}) \backslash \xi$. And Lemma 7.9 proves $\sigma=k \mathbb{Z} \subseteq d \mathbb{Z}=\{0\}$, implying $k=0$ and thus $\sigma=\{0\}$. As, naturally, $E \subseteq\{0\} \cup \mathbb{N}$, the tuple $(\sigma, \lambda, \kappa, \xi)$ is indeed as claimed in the fifth row of the table.

Case 1.2: Should $d \geq 1$ on the other hand, then by Lemma 7.12 (c) we infer $\xi=\mathbb{Z} \backslash D_{d}$ for $D:=\left(\{0\} \cup \mathbb{I}\left\lfloor\frac{d}{2}\right\rfloor \rrbracket\right) \backslash \xi$. Since $\sigma=k \mathbb{Z} \subseteq d \mathbb{Z}$ by Lemma 7.9, if we put $u:=\frac{k}{d}$, then $\sigma=u d \mathbb{Z}$. Recognizing $D \subseteq\{0\} \cup \llbracket\left\lfloor\frac{d}{2}\right\rfloor \rrbracket$ and defining $m:=d$ thus proves that $(\sigma, \lambda, \kappa, \xi)$ is as asserted by the third row of the table.

Case 2: Now, let $\lambda \neq \varnothing$ instead. Then, $(\lambda, \kappa)=(l+2 l \mathbb{Z}, 2 l \mathbb{Z})$ or $(\lambda, \kappa)=(l \mathbb{Z}, l \mathbb{Z})$ by Lemma 7.7 (a). Respectively, $d=2 l$ or $d=l$. We now distinguish two cases based on the value of $l \in\{0\} \cup \mathbb{N}$.

Case 2.1: Assuming $l=0$ lets us conclude $l \mathbb{Z}=2 l \mathbb{Z}=l+2 l \mathbb{Z}=\{0\}$, which implies $(\lambda, \kappa)=(\{0\},\{0\})$. Lemma 7.9 gives $\sigma=k \mathbb{Z} \subseteq \kappa=\{0\}$ and thus $k=0$ and $\sigma=\{0\}$. Because $d=l=2 l=0$ we can infer $\xi=\mathbb{Z} \backslash E_{0}$ for $E:=(\{0\} \cup \mathbb{N}) \backslash \xi$ by Lemma 7.12 (a). As $E \subseteq\{0\} \cup \mathbb{N}$, the tuple $(\sigma, \lambda, \kappa, \xi)$ is hence given by the fourth row of the table.

Case 2.2: Finally, let $l \geq 0$. Then, also $d \geq 0$, no matter whether $d=l$ or $d=2 l$. In conclusion, $\xi=\mathbb{Z} \backslash D_{l}$ for $\bar{D}:=\left(\{0\} \cup \llbracket\left\lfloor\frac{l}{2}\right\rfloor \rrbracket\right) \backslash \bar{\xi}$ by Lemma 7.12 (c). 
Case 2.2.1: If $(\lambda, \kappa)=(l+2 l \mathbb{Z}, 2 l \mathbb{Z})$, i.e., $d=2 l$, then the implication $\sigma=k \mathbb{Z} \subseteq$ $d \mathbb{Z}=2 l \mathbb{Z}$ of Lemma 7.9 lets us define $u \in\{0\} \cup \mathbb{N}$ by $u:=\frac{k}{2 l}$ and obtain $\sigma=2 u l \mathbb{Z}$. Hence, choosing $m:=l$ proves that $(\sigma, \lambda, \kappa, \xi)$ fits the second row of the table.

Case 2.2.2: If instead, $(\lambda, \kappa)=(l \mathbb{Z}, l \mathbb{Z})$, i.e., $d=l$, then Lemma 7.9 yields $\sigma=k \mathbb{Z} \subseteq$ $d \mathbb{Z}=l \mathbb{Z}$, thus permitting us to define $u \in\{0\} \cup \mathbb{N}$ by $u:=\frac{k}{l}$ and obtain $\sigma=u l \mathbb{Z}$. The choice $m:=l$ hence shows $(\sigma, \lambda, \kappa, \xi)$ to be given by the first row.

As mentioned before, our goal will be to show (Section 7.3) that for every nonhyperoctahedral category $\mathcal{C} \subseteq \mathcal{P}^{\circ}$ the tuple $(\Sigma, L, K, X)(\mathcal{C})$ is of the form given in the table of the Arithmetic Lemma.

\subsection{Reduction to Singleton and Pair Blocks}

Let us return to categories of partitions. To elucidate the ranges of $K, L$ and $X$ over $P C a t_{\mathrm{NHO}}^{\circ \bullet}$ and central relations between $\Sigma(\mathcal{C}), K(\mathcal{C}), L(\mathcal{C})$ and $X(\mathcal{C})$ for non-hyperoctahedral categories $\mathcal{C} \subseteq \mathcal{P}^{\circ}$, we must consider certain decompositions of $K, L$ and $X$ according to leg colors.

Definition 7.14 Let $\mathcal{S} \subseteq \mathcal{P}^{\circ}$ and $c_{1}, c_{2} \in\{\circ, \bullet\}$ be abitrary. Then, define

$$
\begin{aligned}
K_{c_{1}, c_{2}}(\mathcal{S}):=\left\{\delta_{p}\left(\alpha_{1}, \alpha_{2}\right) \mid\right. & p \in \mathcal{S}, B \text { block of } p, \alpha_{1}, \alpha_{2} \in B, \alpha_{1} \neq \alpha_{2}, \\
& ] \alpha_{1}, \alpha_{2}\left[{ }_{p} \cap B=\varnothing, \forall i=1,2: \alpha_{i} \text { of normalized color } c_{i}\right\}, \\
X_{c_{1}, c_{2}}(\mathcal{S}):=\left\{\delta_{p}\left(\alpha_{1}, \alpha_{2}\right) \mid\right. & p \in \mathcal{S}, B_{1}, B_{2} \text { blocks of } p, B_{1} \text { and } B_{2} \text { cross, } \\
& \left.\alpha_{1} \in B_{1}, \alpha_{2} \in B_{2}, \forall i=1,2: \alpha_{i} \text { of normalized color } c_{i}\right\} .
\end{aligned}
$$

$L, K$ and $X$ can then be written as, where the union occurs pointwise,

$$
L=\bigcup_{\substack{c_{1}, c_{2} \in\{0, \bullet\} \\ c_{1}=c_{2}}} K_{c_{1}, c_{2}}, \quad K=\bigcup_{\substack{c_{1}, c_{2} \in\{0, \bullet\} \\ c_{1} \neq c_{2}}} K_{c_{1}, c_{2}}, \quad \text { and } X=\bigcup_{c_{1}, c_{2} \in\{0, \bullet\}} X_{c_{1}, c_{2}} .
$$

Recall that $\mathcal{P}_{<2}^{\circ \bullet}$ denotes the set of all partitions with block sizes one or two and that it is a category (see $[\overline{5}$, Lemma 4.4 (a)]). By the next lemma we may always restrict to partitions in $\mathcal{P}_{\leq 2}^{\circ \bullet}$ when studying $K_{c_{1}, c_{2}}$ and $X_{c_{1}, c_{2}}$. This is trivial in cases $\mathcal{O}$ and $\mathcal{B}$, while for case $\mathcal{S}$ this basically follows from Lemma 4.1(c).

Lemma 7.15 For all non-hyperoctahedral categories $\mathcal{C} \subseteq \mathcal{P}^{\circ}$ and $c_{1}, c_{2} \in\{\circ, \bullet\}$ :

(a) $K_{c_{1}, c_{2}}(\mathcal{C})=K_{c_{1}, c_{2}}\left(\mathcal{C} \cap \mathcal{P}_{\leq 2}^{\circ \bullet}\right)$.

(b) $X_{c_{1}, c_{2}}(\mathcal{C})=X_{c_{1}, c_{2}}\left(\mathcal{C} \cap \mathcal{P}_{\leq 2}^{0 \bullet}\right)$.

Proof (a) If $\mathcal{C}$ is case $\mathcal{O}$ or case $\mathcal{B}$, i.e., if $\mathcal{C} \subseteq \mathcal{P}_{\leq 2}^{\circ \bullet}$ by Proposition 4.3, there is nothing to show. Hence, suppose that $\mathcal{C}$ is case $\mathcal{S}$ and let $c_{1}, c_{2} \in\{0, \bullet\}$. We only need to prove $K_{c_{1}, c_{2}}(\mathcal{C}) \subseteq K_{c_{1}, c_{2}}\left(\mathcal{C} \cap \mathcal{P}_{\leq 2}^{\circ}\right)$. Let $\alpha_{1}$ and $\alpha_{2}$ with $\alpha_{1} \neq \alpha_{2}$ be points in $p \in \mathcal{C}$ such that $\alpha_{i}$ is of normalized color $c_{i}$ for every $i \in\{1,2\}$ and such that $\alpha_{1}, \alpha_{2} \in B$ and ]$\alpha_{1}, \alpha_{2}\left[{ }_{p} \cap B=\varnothing\right.$ for some block $B$ in $p$. Because $\mathcal{C}$ is case $\mathcal{S}$, by Lemma 4.1 (c) we do not violate the assumption $p \in \mathcal{C}$ by assuming that every block other than $B$ is a singleton. In the same way we can assume that $\alpha_{1}$ and $\alpha_{2}$ are the only legs of $B$. None of these assumptions affect $\delta_{p}\left(\alpha_{1}, \alpha_{2}\right)$ or the normalized colors of $\alpha_{1}$ or $\alpha_{2}$. As they ensure $p \in \mathcal{C} \cap \mathcal{P}_{\leq 2}^{\circ \bullet}$ though, we have shown $\delta_{p}\left(\alpha_{1}, \alpha_{2}\right) \in K_{c_{1}, c_{2}}\left(\mathcal{C} \cap \mathcal{P}_{\leq 2}^{\circ \bullet}\right)$, which is what we needed to see. 
(b) Again, all that we need to prove is that $X_{c_{1}, c_{2}}(\mathcal{C}) \subseteq X_{c_{1}, c_{2}}\left(\mathcal{C} \cap \mathcal{P}_{<2}^{\circ \bullet}\right)$ if $\mathcal{C}$ is case $\mathcal{S}$ and if $c_{1}, c_{2} \in\{\circ, \bullet\}$. Let the points $\alpha_{1}$ of normalized color $c_{1}$ and $\alpha_{2}$ of normalized color $c_{2}$ in $p \in \mathcal{C}$ belong to the blocks $B_{1}$ and $B_{2}$, respectively, and suppose that $B_{1}$ and $B_{2}$ cross. Because $\mathcal{C}$ is case $\mathcal{S}$ we can, by Lemma 4 .1 (c), assume that all other blocks of $p$ besides $B_{1}$ and $B_{2}$ are singletons. Now the only thing standing in the way of $p \in \mathcal{C} \cap \mathcal{P}_{\leq 2}^{\circ \bullet}$ is the possibility of at least one of $B_{1}$ and $B_{2}$ having more than two legs. We would like to assume that $B_{1}$ and $B_{2}$ have only two legs each and still maintain all the other assumptions including $\alpha_{1} \in B_{1}$ and $\alpha_{2} \in B_{2}$ and, of course, not alter $\delta_{p}\left(\alpha_{1}, \alpha_{2}\right)$. By Lemma 4.1 (c), we can always remove surplus legs of $B_{1}$ and $B_{2}$. But it is not immediately clear that we can remove legs without affecting the other assumptions. A priori, the crossing between $B_{1}$ and $B_{2}$ only implies that we can find points $\beta_{1}, \gamma_{1} \in B_{1}$ and $\beta_{2}, \gamma_{2} \in B_{2}$ such that $\left(\beta_{1}, \beta_{2}, \gamma_{1}, \gamma_{2}\right)$ is ordered in $p$. If now $\alpha_{1} \in\left\{\beta_{1}, \gamma_{1}\right\}$ and $\alpha_{2} \in\left\{\beta_{2}, \gamma_{2}\right\}$, then we can certainly remove all legs except $\left\{\beta_{i}, \gamma_{i}\right\}$ from $B_{i}$ for all $i \in\{1,2\}$ and still maintain the other assumptions. In fact, we can do so in general as well:

Let us only consider the "worst case" that $\alpha_{1} \notin\left\{\beta_{1}, \gamma_{1}\right\}$ and $\alpha_{2} \notin\left\{\beta_{2}, \gamma_{2}\right\}$. There are 20 possible arrangements of the points $\left\{\alpha_{1}, \beta_{1}, \gamma_{1}, \alpha_{2}, \beta_{2}, \gamma_{2}\right\}$ relative to each other with respect to the cyclic order respecting that $\left(\beta_{1}, \beta_{2}, \gamma_{1}, \gamma_{2}\right)$ is ordered.

\begin{tabular}{|c|c|c|c|}
\hline$\stackrel{\downarrow}{\alpha} \beta_{1} \beta_{2} \gamma_{1} \gamma_{2}$ & $\beta_{1} \alpha_{1}^{\downarrow} \beta_{2} \gamma_{1} \gamma_{2}$ & $\beta_{1} \beta_{2} \alpha_{1} \gamma_{1} \gamma_{2}$ & $\beta_{1} \beta_{2} \gamma_{1} \alpha_{1} \gamma_{2}$ \\
\hline$\alpha_{2} \alpha_{1} \beta_{1} \beta_{2} \gamma_{1} \gamma_{2}$ & $\alpha_{2} \beta_{1} \alpha_{1} \beta_{2} \gamma_{1} \gamma_{2}$ & $\alpha_{2} \beta_{1} \beta_{2} \alpha_{1} \gamma_{1} \gamma_{2}$ & $\alpha_{2} \beta_{1} \beta_{2} \gamma_{1} \underline{\alpha_{1}} \gamma_{2}$ \\
\hline$\beta_{1} \overline{\beta_{2} \gamma_{1}} \gamma_{2}$ & $\overline{\beta_{1}} \alpha_{2} \overline{\alpha_{1} \beta_{2} \gamma_{1}} \gamma_{2}$ & $\overline{\beta_{1} \alpha_{2} \beta_{2} \alpha_{1}} \gamma_{1} \gamma_{2}$ & $\overline{\beta_{1} \alpha_{2} \beta_{2}} \gamma_{1} \overline{\alpha_{1}} \gamma_{2}$ \\
\hline$\alpha_{1} \beta_{1} \alpha_{2} \beta_{2} \gamma_{1} \gamma_{2}$ & $\beta_{1} \alpha_{1} \alpha_{2} \beta_{2} \gamma_{1} \gamma_{2}$ & $\beta_{1} \beta_{2} \alpha_{2} \alpha_{1} \gamma_{1}$ & $\overline{\beta_{1} \beta_{2}} \alpha_{2} \gamma_{1}$ \\
\hline$\overline{\alpha_{1}} \beta_{1} \overline{\beta_{2}} \alpha_{2} \overline{\gamma_{1} \gamma_{2}}$ & $\beta_{1} \overline{\alpha_{1} \beta_{2}} \alpha_{2} \overline{\gamma_{1} \gamma_{2}}$ & $\overline{\beta_{1}} \beta_{2} \overline{\alpha_{1} \alpha_{2}} \gamma_{1} \overline{\gamma_{2}}$ & $\overline{\beta_{1}} \beta_{2} \overline{\gamma_{1}} \alpha_{2} \overline{\alpha_{1} \gamma_{2}}$ \\
\hline$\overline{\alpha_{1}} \beta_{1} \beta_{2} \overline{\gamma_{1} \alpha_{2} \gamma_{2}}$ & $\beta_{1} \overline{\alpha_{1}} \beta_{2} \overline{\gamma_{1} \alpha_{2} \gamma_{2}} \mid$ & $\beta_{1} \beta_{2} \overline{\alpha_{1} \gamma_{1} \alpha_{2} \gamma_{2}}$ & $\overline{\beta_{1}} \beta_{2} \gamma_{1} \overline{\alpha_{1} \alpha_{2} \gamma_{2}}$ \\
\hline
\end{tabular}

We remove all legs of $B_{1}$ and $B_{2}$ except for the underlined ones. Then the above table shows that we can always turn $B_{1}$ and $B_{2}$ into crossing pair blocks containing $\alpha_{1}$ and $\alpha_{2}$, respectively. That concludes the proof.

\subsection{Verifying the Axioms}

We want to apply the Arithmetic Lemma 7.13 to the sets $\sigma:=\Sigma(\mathcal{C}), \kappa_{c_{1}, c_{2}}:=K_{c_{1}, c_{2}}(\mathcal{C})$ and $\xi_{c_{1}, c_{2}}:=X_{c_{1}, c_{2}}(\mathcal{C})$ for $c_{1}, c_{2} \in\{\circ, \bullet\}$ and non-hyperoctahedral categories $\mathcal{C} \subseteq \mathcal{P}^{\circ} \bullet$ In order to be able to do so, we, of course, need to show that these sets actually satisfy the prerequisite Axioms 7.1. Proving that will crucially utilize the reduction to singleton and pair blocks from Lemma 7.15.

Lemma 7.16 For every non-hyperoctahedral category $\mathcal{C} \subseteq \mathcal{P}^{\circ}$, the set $\sigma:=\Sigma(\mathcal{C})$ satisfies Axiom (i) of 7.1: $\sigma$ is a subgroup of $\mathbb{Z}$.

Proof That was shown in Proposition 6.1.

Lemma 7.17 For every non-hyperoctahedral category $\mathcal{C} \subseteq \mathcal{P}^{\circ}$, the sets $\sigma:=\Sigma(\mathcal{C})$ and $\kappa_{c_{1}, c_{2}}:=K_{c_{1}, c_{2}}(\mathcal{C})$ for $c_{1}, c_{2} \in\{\circ, \bullet\}$ satisfy Axioms (ii)-(iv) of 7.1:
(ii) $\kappa_{c_{1}, c_{2}}+\sigma \subseteq \kappa_{c_{1}, c_{2}}$,
(iii) $\kappa_{c_{1}, c_{2}} \subseteq-\kappa_{\overline{c_{2}}, \overline{c_{1}}}$,
(iv) $\kappa_{c_{1}, c_{2}} \subseteq-\kappa_{c_{2}, c_{1}}+\sigma$

for all $c_{1}, c_{2} \in\{\circ, \bullet\}$. 
Proof Let $c_{1}, c_{2} \in\{\circ, \bullet\}$ be arbitrary and let $\alpha_{1}$ and $\alpha_{2}$ be distinct points of the same block $B$ in $p \in \mathcal{C}$ such that $] \alpha_{1}, \alpha_{2}\left[{ }_{p} \cap B=\varnothing\right.$ and such that $\alpha_{i}$ has normalized color $c_{i}$ for every $i \in\{1,2\}$. In other words, let $\delta_{p}\left(\alpha_{1}, \alpha_{2}\right)$ be a generic element of $K_{c_{1}, c_{2}}(\mathcal{C})=\kappa_{c_{1}, c_{2}}$.

Axiom (ii): Let $q \in \mathcal{C}$ be arbitrary. None of the assumptions about $p, \alpha_{1}, \alpha_{2}$ and $\delta_{p}\left(\alpha_{2}, \alpha_{2}\right)$ are impacted by assuming that $p$ is rotated in such a way that $\alpha_{1}$ is the rightmost lower point of $p$. Then, $B$ is a block of $p \otimes q \in \mathcal{C}$ as well and $] \alpha_{1}, \alpha_{2}[p \otimes q \cap B=\varnothing$.
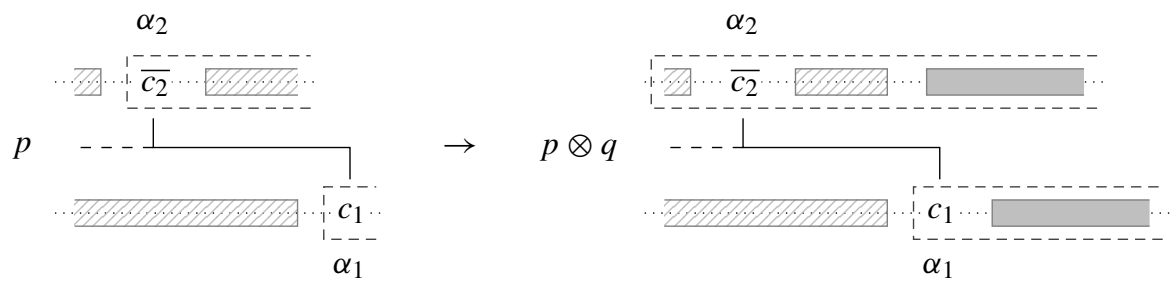

Now, because all points stemming from $q$ lie within $] \alpha_{1}, \alpha_{2}[p \otimes q$,

$$
\delta_{p \otimes q}\left(\alpha_{1}, \alpha_{2}\right)=\delta_{p}\left(\alpha_{1}, \alpha_{2}\right)+\Sigma(q) .
$$

That proves $\delta_{p}\left(\alpha_{1}, \alpha_{2}\right)+\Sigma(q) \in K_{c_{1}, c_{2}}(\mathcal{C})=\kappa_{c_{1}, c_{2}}$, which is what we needed to see.

Axiom (iii): The verticolor reflection $\tilde{p}$ of $p$ belongs to $\mathcal{C}$. The set $\left.] \alpha_{1}, \alpha_{2}\right]_{p}$ in $p$ is mapped by the reflection $\rho$ to the set $\left[\rho\left(\alpha_{2}\right), \rho\left(\alpha_{1}\right)[\tilde{p}\right.$ in $\tilde{p}$. As the operation of verticolor reflection inverts normalized colors, $\sigma_{p}(S)=-\sigma_{\tilde{p}}(\rho(S))$ for any set $S$ of points in $p$.

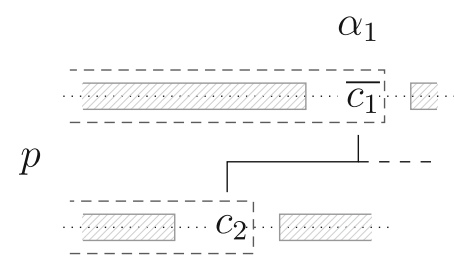

$\alpha_{2}$

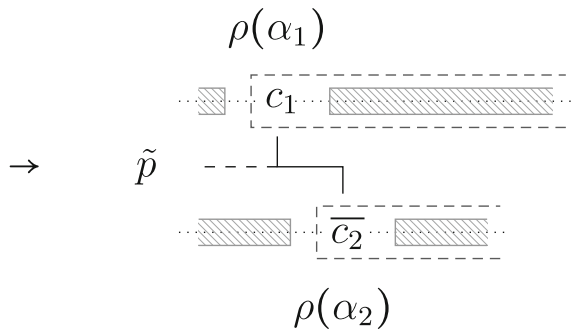

Using the case distinction free formula for $\delta_{p}\left(\alpha_{1}, \alpha_{2}\right)$ given in the proof of [5, Lemma 3.1 (b)], we thus compute

$$
\begin{aligned}
\delta_{p}\left(\alpha_{1}, \alpha_{2}\right)= & \left.\left.\sigma_{p}(] \alpha_{1}, \alpha_{2}\right]_{p}\right)+\frac{1}{2}\left(\sigma_{p}\left(\alpha_{1}\right)-\sigma_{p}\left(\alpha_{2}\right)\right) \\
= & -\sigma_{\tilde{p}}\left(\left[\rho\left(\alpha_{2}\right), \rho\left(\alpha_{1}\right)[\tilde{p})-\frac{1}{2}\left(\sigma_{\tilde{p}}\left(\rho\left(\alpha_{1}\right)\right)-\sigma_{\tilde{p}}\left(\rho\left(\alpha_{2}\right)\right)\right)\right.\right. \\
= & \left.\left.-\sigma_{\tilde{p}}(] \rho\left(\alpha_{2}\right), \rho\left(\alpha_{1}\right)\right]_{\tilde{p}}\right)-\sigma_{\tilde{p}}\left(\rho\left(\alpha_{2}\right)\right)+\sigma_{\tilde{p}}\left(\rho\left(\alpha_{1}\right)\right) \\
& -\frac{1}{2}\left(\sigma_{\tilde{p}}\left(\rho\left(\alpha_{1}\right)\right)-\sigma_{\tilde{p}}\left(\rho\left(\alpha_{2}\right)\right)\right) \\
= & \left.\left.-\sigma_{\tilde{p}}(] \rho\left(\alpha_{2}\right), \rho\left(\alpha_{1}\right)\right]_{\tilde{p}}\right)-\frac{1}{2}\left(\sigma_{\tilde{p}}\left(\rho\left(\alpha_{2}\right)\right)-\sigma_{\tilde{p}}\left(\rho\left(\alpha_{1}\right)\right)\right) \\
= & -\delta_{\tilde{p}}\left(\rho\left(\alpha_{2}\right), \rho\left(\alpha_{1}\right)\right) .
\end{aligned}
$$

Because, for every $i \in\{1,2\}$, the point $\rho\left(\alpha_{i}\right)$ has normalized color $\overline{c_{i}}$ in $\tilde{p}$ and because $\rho(B)$ is a block of $\tilde{p}$ with $] \rho\left(\alpha_{2}\right), \rho\left(\alpha_{1}\right)\left[\tilde{p} \cap \rho(B)=\varnothing\right.$, we conclude $\delta_{p}\left(\alpha_{1}, \alpha_{2}\right) \in-K_{\overline{c_{2}}, \overline{c_{1}}}(\mathcal{C})=$ $-\kappa_{\overline{c_{2}}}, \overline{c_{1}}$. And that is what we had to show.

Axiom (iv): So far, we have not made use of Lemma 7.15. Now, though, we employ it to additionally assume $p \in \mathcal{C} \cap \mathcal{P}_{\leq 2}^{\circ}$. In particular, then, $B=\left\{\alpha_{1}, \alpha_{2}\right\}$ is a pair block. Consequently, not only $] \alpha_{1}, \alpha_{2}\left[{ }_{p} \cap B=\varnothing\right.$ but also $] \alpha_{2}, \alpha_{1}\left[{ }_{p} \cap B=\varnothing\right.$. 

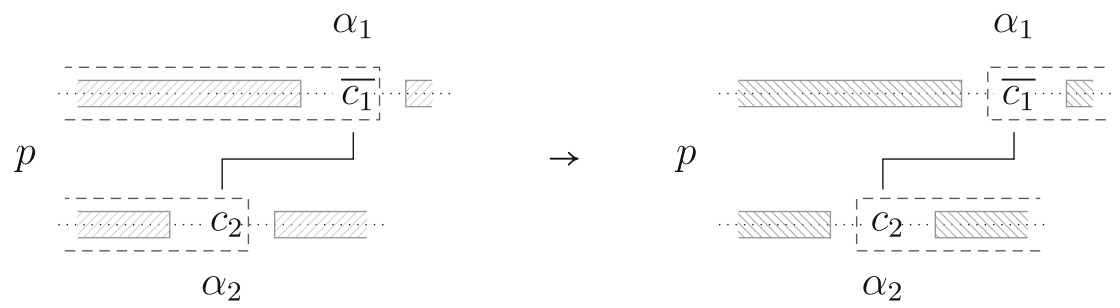

By [5, Lemma 2.1 (b)] we infer

$$
\delta_{p}\left(\alpha_{1}, \alpha_{2}\right)=-\delta_{p}\left(\alpha_{2}, \alpha_{1}\right) \bmod \Sigma(p) .
$$

As $\Sigma(p) \in \Sigma(\mathcal{C})$, it follows $\delta_{p}\left(\alpha_{1}, \alpha_{2}\right) \in-K_{c_{2}, c_{1}}(\mathcal{C})+\Sigma(\mathcal{C})=-\kappa_{c_{2}, c_{1}}+\sigma$, which is what we wanted to see.

Lemma 7.18 For every non-hyperoctahedral category $\mathcal{C} \subseteq \mathcal{P}^{\circ}$, the sets $\sigma:=\Sigma(\mathcal{C})$ and $\xi_{c_{1}, c_{2}}:=X_{c_{1}, c_{2}}(\mathcal{C})$ for $c_{1}, c_{2} \in\{\circ, \bullet\}$ satisfy Axioms (ii)-(iv) of 7.1:
(ii) $\xi_{c_{1}, c_{2}}+\sigma \subseteq \xi_{c_{1}, c_{2}}$,
(iii) $\xi_{c_{1}, c_{2}} \subseteq-\xi_{\overline{c_{2}}, \overline{c_{1}}}$,
(iv) $\xi_{c_{1}, c_{2}} \subseteq-\xi_{c_{2}, c_{1}}+\sigma$

for all $c_{1}, c_{2} \in\{\circ, \bullet\}$.

Proof The proof is similar to that of Lemma 7.17. Let $c_{1}, c_{2} \in\{\circ, \bullet\}$, let $B_{1}$ and $B_{2}$ be crossing blocks of $p \in \mathcal{C}$ and let $\alpha_{1} \in B_{1}$ and $\alpha_{2} \in B_{2}$ have normalized colors $c_{1}$ and $c_{2}$, respectively. That makes $\delta_{p}\left(\alpha_{1}, \alpha_{2}\right)$ a generic element of $X_{c_{1}, c_{2}}(\mathcal{C})=\xi_{c_{1}, c_{2}}$.

Axiom (ii): Just like in the proof of Lemma 7.17, we can assume that $\alpha_{1}$ is the rightmost lower point. Given arbitrary $q \in \mathcal{C}$, the sets $B_{1}$ and $B_{2}$ are crossing blocks of $p \otimes q$ as well,
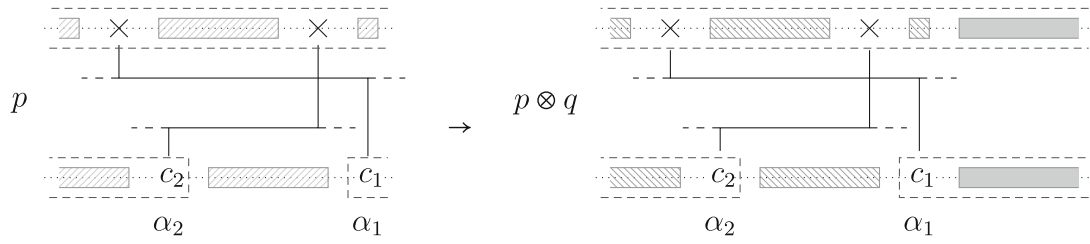

which proves

$$
\delta_{p}\left(\alpha_{1}, \alpha_{2}\right)+\Sigma(q)=\delta_{p \otimes q}\left(\alpha_{1}, \alpha_{2}\right) \in X_{c_{1}, c_{2}}(\mathcal{C})=\xi_{c_{1}, c_{2}} .
$$

Thus, $\xi_{c_{1}, c_{2}}+\sigma \subseteq \xi_{c_{1}, c_{2}}$ as claimed.

Axiom (iii): Likewise, the sets $\rho\left(B_{1}\right)$ and $\rho\left(B_{2}\right)$ are still crossing blocks in $\tilde{p} \in \mathcal{C}$. There, $\alpha_{i}$ has normalized color $\overline{c_{i}}$ for every $i \in\{1,2\}$.
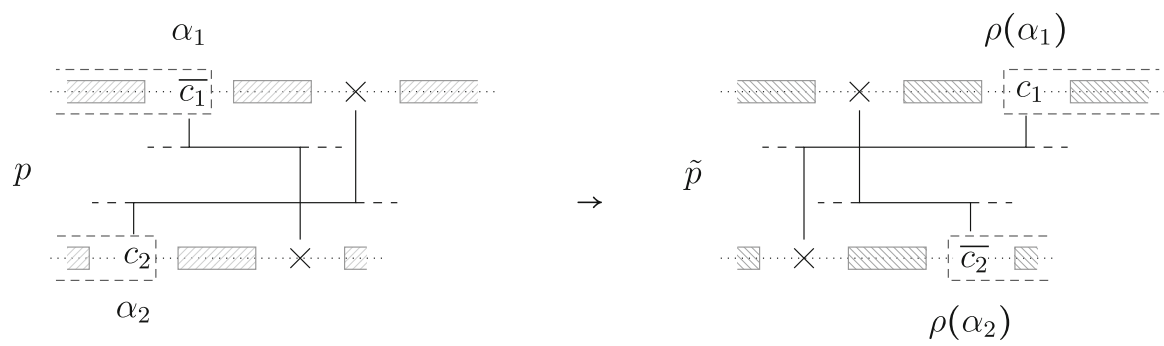

and because, $\delta_{p}\left(\alpha_{1}, \alpha_{2}\right) \equiv-\delta_{p}\left(\alpha_{2}, \alpha_{1}\right) \bmod \Sigma(p)$ (by [5, Lemma 2.1 (b)]), we can immediately conclude $\delta_{p}\left(\alpha_{1}, \alpha_{2}\right) \in-X_{c_{2}, c_{1}}(\mathcal{C})+\Sigma(\mathcal{C})$. Thus, $\xi_{c_{1}, c_{2}} \subseteq-\xi_{c_{2}, c_{1}}+\sigma$. Differently from Lemma 7.17, we did not need Lemma 7.15 to see this. 
Lemma 7.19 For every non-hyperoctahedral category $\mathcal{C} \subseteq \mathcal{P}^{\circ \bullet}$, the sets $\sigma:=\Sigma(\mathcal{C})$ and $\xi_{c_{1}, c_{2}}:=X_{c_{1}, c_{2}}(\mathcal{C})$ for $c_{1}, c_{2} \in\{\circ, \bullet\}$ satisfy Axiom (v) of 7.1: For all $c_{1}, c_{2} \in\{\circ, \bullet\}$,

$$
\xi_{c_{1}, c_{2}} \subseteq \xi_{c_{1}, \overline{c_{2}}} \cup\left(-\xi_{c_{2}, \overline{c_{1}}}+\sigma\right)
$$

Proof Let $B_{1}$ and $B_{2}$ be crossing blocks in $p \in \mathcal{C} \cap \mathcal{P}_{\leq 2}^{\circ \bullet}$ and let $\alpha_{1} \in B_{1}$ and $\alpha_{2} \in B_{2}$ have normalized colors $c_{1} \in\{\circ, \bullet\}$ and $c_{2} \in\{\circ, \bullet\}$, respectively. According to Lemma 7.15 then, every element of $\xi_{c_{1}, c_{2}}=X_{c_{1}, c_{2}}(\mathcal{C})=X_{c_{1}, c_{2}}\left(\mathcal{C} \cap \mathcal{P}_{\leq 2}^{\circ}\right)$ is of the form $\delta_{p}\left(\alpha_{1}, \alpha_{2}\right)$. Because $p \in \mathcal{P}_{\leq 2}^{\circ}$, the blocks $B_{1}$ and $B_{2}$ are pairs. Hence, the crossing between these blocks means that we find points $\beta_{1} \in B_{1}$ and $\beta_{2} \in B_{2}$ with $\alpha_{1} \neq \beta_{1}$ and $\alpha_{2} \neq \beta_{2}$ such that either $\left(\alpha_{1}, \alpha_{2}, \beta_{1}, \beta_{2}\right)$ or $\left(\alpha_{2}, \alpha_{1}, \beta_{2}, \beta_{1}\right)$ is ordered.

Case 1: First, we suppose that $\left(\alpha_{1}, \alpha_{2}, \beta_{1}, \beta_{2}\right)$ is ordered and show $\delta_{p}\left(\alpha_{1}, \alpha_{2}\right) \in X_{c_{1}, \overline{c_{2}}}(\mathcal{C})$. We can assume that $\alpha_{1}$ is the leftmost and $\beta_{1}$ the rightmost lower point.

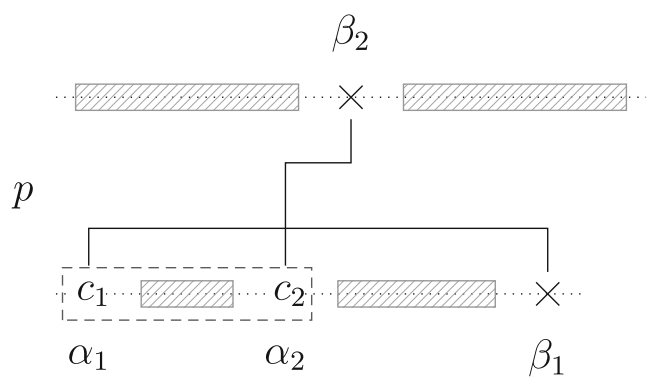

By Lemma 3.3, the partition $p^{\prime}:=P\left(p,\left[\alpha_{1}, \beta_{1}\right]_{p}\right)$ belongs to $\mathcal{C}$. The definition of the projection operation has the following consequences: The three lower points $\alpha_{1}, \alpha_{2}$ and $\beta_{1}$ of $p$, also points of $p^{\prime}$, all retain their normalized colors in $p^{\prime}$; the set $B_{1}=\left\{\alpha_{1}, \beta_{1}\right\}$ is still a block of $p^{\prime}$; the point $\alpha_{2}$ is now connected to its counterpart $\beta_{2}^{\prime}$ on the upper row of $p^{\prime}$, implying in particular that the blocks of $\alpha_{1}$ and $\alpha_{2}$ still cross in $p^{\prime}$; and it holds

$$
\delta_{p^{\prime}}\left(\alpha_{1}, \alpha_{2}\right)=\delta_{p}\left(\alpha_{1}, \alpha_{2}\right)
$$

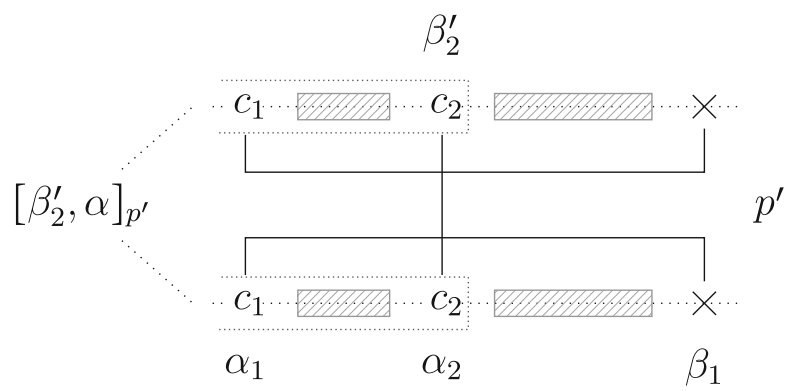

We apply Lemma 3.3 a second time to infer $p^{\prime \prime}:=P\left(p^{\prime},\left[\beta_{2}^{\prime}, \alpha_{2}\right]\right) \in \mathcal{C}$. Denote the images of the points $\beta_{2}^{\prime}, \alpha_{1}$ and $\alpha_{2}$ of $p^{\prime}$ in $p^{\prime \prime}$ by $\beta_{2}^{\prime \prime}, \alpha_{1}^{\prime \prime}$ and $\alpha_{2}^{\prime \prime}$, respectively. Now, $\beta_{2}^{\prime \prime}$ is the leftmost lower point and $\alpha_{2}^{\prime \prime}$ the rightmost lower point of $p^{\prime \prime}$ and the two form a block; the point $\alpha_{1}^{\prime \prime} \in\left[\beta_{2}^{\prime \prime}, \alpha_{2}^{\prime \prime}\right]_{p^{\prime \prime}}$ is connected to its counterpart on the upper row; and

$$
\delta_{p^{\prime \prime}}\left(\alpha_{1}^{\prime \prime}, \alpha_{2}^{\prime \prime}\right)=\delta_{p^{\prime}}\left(\alpha_{1}, \alpha_{2}\right)=\delta_{p}\left(\alpha_{1}, \alpha_{2}\right)
$$




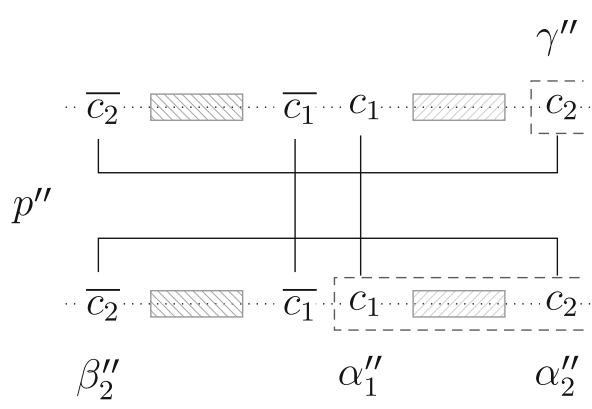

There are two crucial observations to make about the successor $\gamma^{\prime \prime}$ of $\alpha_{2}^{\prime \prime}$ in $p^{\prime \prime}$, the rightmost upper point of $p^{\prime \prime}$. Firstly, $\gamma^{\prime \prime}$ has the inverse normalized color $\overline{c_{2}}$ of $\alpha_{2}^{\prime \prime}$, which in particular implies that $\delta_{p^{\prime \prime}}\left(\alpha_{2}^{\prime \prime}, \gamma^{\prime \prime}\right)=0$. Secondly, $\gamma^{\prime \prime}$ forms a block of $p^{\prime \prime}$ together with the leftmost upper point of $p^{\prime \prime}$, which entails that its block crosses the block of $\alpha_{1}^{\prime \prime}$ in $p^{\prime \prime}$. Hence, $\delta_{p^{\prime \prime}}\left(\alpha_{1}^{\prime \prime}, \gamma^{\prime \prime}\right) \in X_{c_{1}, \overline{c_{2}}}(\mathcal{C})$ and

$$
\delta_{p^{\prime \prime}}\left(\alpha_{1}^{\prime \prime}, \gamma^{\prime \prime}\right)=\delta_{p^{\prime \prime}}\left(\alpha_{1}^{\prime \prime}, \alpha_{2}^{\prime \prime}\right)+\delta_{p^{\prime \prime}}\left(\alpha_{2}^{\prime \prime}, \gamma^{\prime \prime}\right)=\delta_{p}\left(\alpha_{1}, \alpha_{2}\right)
$$

together show $\delta_{p}\left(\alpha_{1}, \alpha_{2}\right) \in X_{c_{1}, \overline{c_{2}}}(\mathcal{C})=\xi_{c_{1}, \overline{c_{2}}}$, which is what we set out to prove.

Case 2: Now, let $\left(\alpha_{2}, \alpha_{1}, \beta_{2}, \beta_{1}\right)$ be ordered instead. By Case 1 then, $\delta_{p}\left(\alpha_{2}, \alpha_{1}\right) \in$ $X_{c_{2}, \overline{c_{1}}}(\mathcal{C})$. [5, Lemma 2.1 (b)] shows $\delta_{p}\left(\alpha_{2}, \alpha_{1}\right) \equiv-\delta_{p}\left(\alpha_{1}, \alpha_{2}\right) \bmod \Sigma(p)$. That implies $\delta_{p}\left(\alpha_{1}, \alpha_{2}\right) \in-X_{c_{2}, \overline{c_{1}}}(\mathcal{C})+\Sigma(\mathcal{C})=-\xi_{c_{2}, \overline{c_{1}}}+\sigma$, which is what we needed to see.

Lemma 7.20 For every non-hyperoctahedral category $\mathcal{C} \subseteq \mathcal{P}^{\circ}$, the sets $\sigma:=\Sigma(\mathcal{C})$ and $\kappa_{c_{1}, c_{2}}:=K_{c_{1}, c_{2}}(\mathcal{C})$ for $c_{1}, c_{2} \in\{\circ, \bullet\}$ satisfy Axiom (vi) of 7.1: $0 \in \kappa_{\circ} \cap \kappa_{\bullet}$.

Proof Since $\square \in \mathcal{C}$ and $K_{\circ}(\{\lceil\rceil\})=K_{\bullet \circ}(\{\varnothing\})=\{0\}$, this is clear.

Lemma 7.21 For every non-hyperoctahedral category $\mathcal{C} \subseteq \mathcal{P}^{\circ}$, the sets $\sigma:=\Sigma(\mathcal{C})$ and $\kappa_{c_{1}, c_{2}}:=K_{c_{1}, c_{2}}(\mathcal{C})$ for $c_{1}, c_{2} \in\{\circ, \bullet\}$ satisfy Axiom (vii) of 7.1 :

$$
\kappa_{c_{1}, c_{2}}+\kappa_{\overline{c_{2}}, c_{3}} \subseteq \kappa_{c_{1}, c_{3}}
$$

for all $c_{1}, c_{2}, c_{3} \in\{\circ, \bullet\}$.

Proof Let $c_{1}, c_{2}, c_{3} \in\{\circ, \bullet\}$ be arbitrary and let $\eta_{1}$ and $\eta_{2}$ be distinct points of the same block $B$ of $p \in \mathcal{C}$ such that $] \eta_{1}, \eta_{2}\left[{ }_{p} \cap B=\varnothing\right.$ and such that $\eta_{i}$ has normalized color $c_{i}$ in $p$ for every $i \in\{1,2\}$. Furthermore, let $\theta_{1}$ and $\theta_{2}$ be distinct points of the same block $C$ of $q \in \mathcal{C}$ with $] \theta_{1}, \theta_{2}\left[{ }_{q} \cap C=\varnothing\right.$ such that $\theta_{1}$ has normalized color $\overline{c_{2}}$ in $q$ and $\theta_{2}$ normalized color $c_{3}$. None of these assumptions are impacted and neither $\delta_{p}\left(\eta_{1}, \eta_{2}\right)$ nor $\delta_{q}\left(\theta_{1}, \theta_{2}\right)$ altered by assuming that $\eta_{2}$ is the rightmost lower point of $p$ and $\theta_{1}$ the leftmost lower point of $q$.
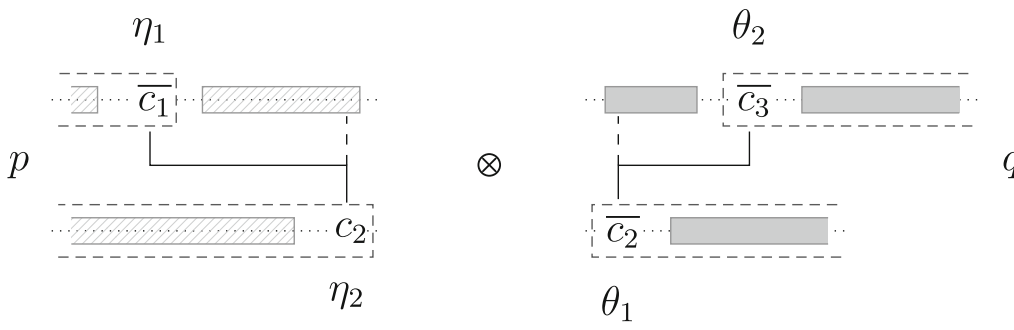

Denote the images of the points $\theta_{1}$ and $\theta_{2}$ of $q$ in $p \otimes q \in \mathcal{C}$ by $\theta_{1}^{\prime}$ and $\theta_{2}^{\prime}$, respectively. The assumptions about the normalized colors of $\eta_{2}$ and $\theta_{1}$ imply that $T:=\left\{\eta_{2}, \theta_{1}^{\prime}\right\}$ is a turn in $p \otimes q$, meaning in particular $\delta_{p \otimes q}\left(\eta_{2}, \theta_{1}^{\prime}\right)=0$. 


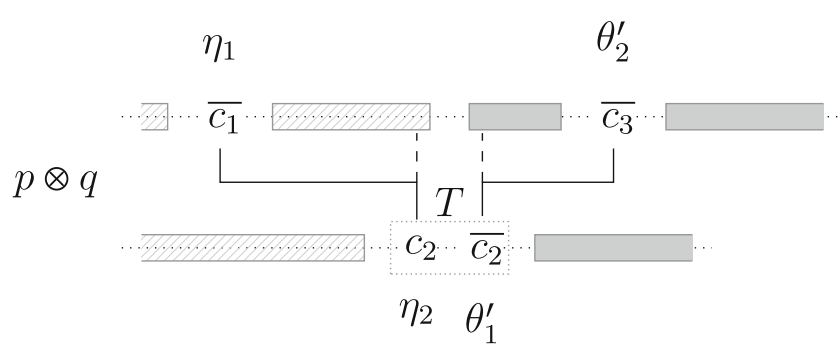

Moreover, $\delta_{p \otimes q}\left(\eta_{1}, \eta_{2}\right)=\delta_{p}\left(\eta_{1}, \eta_{2}\right)$ and $\delta_{p \otimes q}\left(\theta_{1}^{\prime}, \theta_{2}^{\prime}\right)=\delta_{q}\left(\theta_{1}, \theta_{2}\right)$ by nature of the tensor product.

Let $\theta_{2}^{\prime \prime}$ denote the image of $\theta_{2}^{\prime}$ in $r:=E(p \otimes q, T) \in \mathcal{C}$, the partition obtained from $p \otimes q$ by erasing the turn $T$ (see [5, Section 4.3]). By definition of the erasing operation, $\eta_{1}$ and $\theta_{2}^{\prime \prime}$ belong to the same block $D$ in $r$ with $] \eta_{1}, \theta_{2}^{\prime \prime}[r \cap D=\varnothing$.

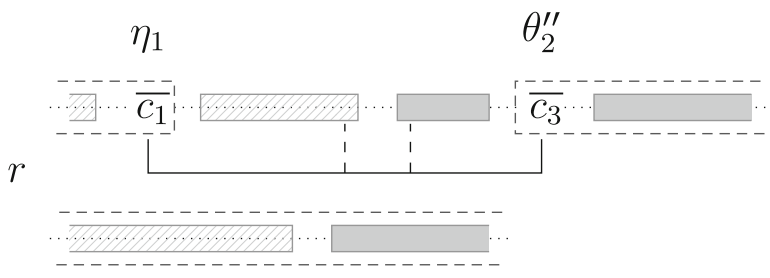

Hence, from $\delta_{r}\left(\eta_{1}, \theta_{2}^{\prime \prime}\right) \in K_{c_{1}, c_{3}}(\mathcal{C})=\kappa_{c_{1}, c_{3}}$ and from

$$
\begin{aligned}
\delta_{r}\left(\eta_{1}, \theta_{2}^{\prime \prime}\right) & =\delta_{p \otimes q}\left(\eta_{1}, \theta_{2}\right)-\sigma_{p \otimes q}(T) \\
& =\delta_{p \otimes q}\left(\eta_{1}, \theta_{2}\right) \\
& =\delta_{p \otimes q}\left(\eta_{1}, \eta_{2}\right)+\delta_{p \otimes q}\left(\eta_{2}, \theta_{1}^{\prime}\right)+\delta_{p \otimes q}\left(\theta_{1}^{\prime}, \theta_{2}^{\prime}\right) \\
& =\delta_{p \otimes q}\left(\eta_{1}, \eta_{2}\right)+\delta_{p \otimes q}\left(\theta_{1}^{\prime}, \theta_{2}^{\prime}\right) \\
& =\delta_{p}\left(\eta_{1}, \eta_{2}\right)+\delta_{q}\left(\theta_{1}, \theta_{2}\right)
\end{aligned}
$$

it follows $\delta_{p}\left(\eta_{1}, \eta_{2}\right)+\delta_{q}\left(\theta_{1}, \theta_{2}\right) \in \kappa_{c_{1}, c_{3}}$. And that is what we needed to show.

Lemma 7.22 For every non-hyperoctahedral category $\mathcal{C} \subseteq \mathcal{P}^{\circ \bullet}$, the sets $\sigma:=\Sigma(\mathcal{C})$ and $\kappa_{c_{1}, c_{2}}:=K_{c_{1}, c_{2}}(\mathcal{C})$ and $\xi_{c_{1}, c_{2}}:=X_{c_{1}, c_{2}}(\mathcal{C})$ for $c_{1}, c_{2} \in\{\circ, \bullet\}$ satisfy Axiom (viii) of 7.1: For all $c_{1}, c_{2}, c_{3} \in\{\circ, \bullet\}$,

$$
\kappa_{c_{1}, c_{2}}+\xi_{\overline{c_{2}}, c_{3}} \subseteq \xi_{c_{1}, c_{3}} .
$$

Proof We adapt the proof of Lemma 7.21. Let $c_{1}, c_{2} \in\{\circ, \bullet\}$ be arbitrary. Let $p, q \in \mathcal{C}$, let $B$ be a block in $p$, and let $C$ and $D$ be two crossing blocks in $q$. Let $\gamma_{1}$ and $\gamma_{2}$ be two distinct points of $B$ of normalized colors $c_{1}$ respectively $c_{2}$ in $p$ with $] \gamma_{1}, \gamma_{2}\left[{ }_{p} \cap B=\varnothing\right.$. In $q$, let $\eta_{1} \in C$ have normalized color $\overline{c_{2}}$ and $\theta_{1} \in D$ normalized color $c_{3}$. Then, $\delta_{p}\left(\gamma_{1}, \gamma_{2}\right)$ is a generic element of $K_{c_{1}, c_{2}}(\mathcal{C})=\kappa_{c_{1}, c_{2}}$ and $\delta_{q}\left(\eta_{1}, \theta_{1}\right)$ one of $X_{\overline{c_{2}}, c_{3}}(\mathcal{C})=\xi_{\overline{c_{2}}, c_{3}}$. No generality is lost assuming that $\gamma_{2}$ is the rightmost lower point of $p$ and $\eta_{1}$ the leftmost lower point of $q$. We find $\eta_{2} \in C$ and $\theta_{2} \in D$ such that $\eta_{1} \neq \eta_{2}$ and $\theta_{1} \neq \theta_{2}$ and such that $\left(\eta_{1}, \theta_{1}, \eta_{2}, \theta_{2}\right)$ or $\left(\eta_{1}, \theta_{2}, \eta_{2}, \theta_{1}\right)$ is ordered in $q$. 

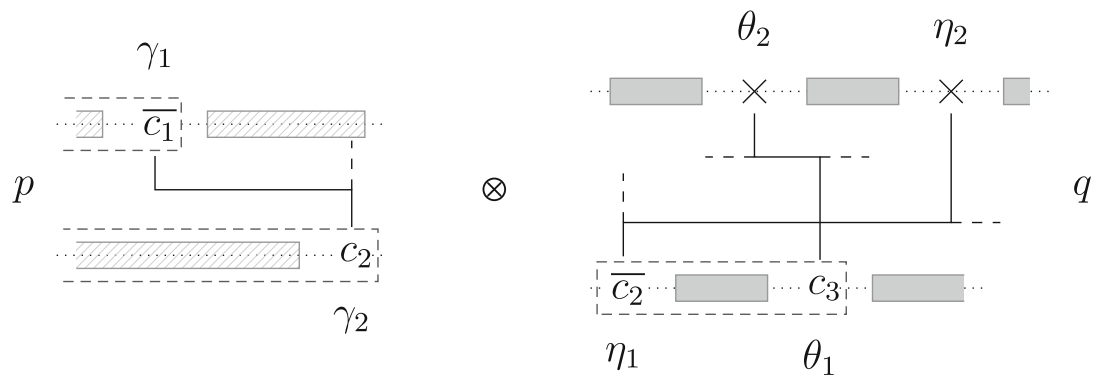

Let $\eta_{1}^{\prime}, \eta_{2}^{\prime}, \theta_{1}^{\prime}$ and $\theta_{2}^{\prime}$ denote the images of, respectively, $\eta_{1}, \eta_{2}, \theta_{1}$ and $\theta_{2}$ in $p \otimes q \in \mathcal{C}$. By nature of the tensor product, $B$ is a block of $p \otimes q$. Likewise, $\eta_{1}^{\prime}$ and $\eta_{2}^{\prime}$ belong to the same block in $p \otimes q$ and so do $\theta_{1}^{\prime}$ and $\theta_{2}^{\prime}$. And each involved point has the same normalized color in $p \otimes q$ as the corresponding preimage in $p$ or $q$. The set $T:=\left\{\gamma_{2}, \eta_{1}^{\prime}\right\}$ is a turn in $p \otimes q$.

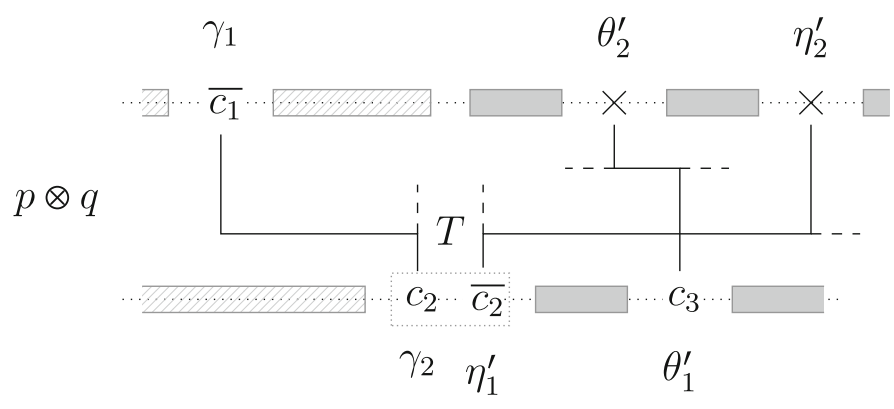

If we denote by $\eta_{2}^{\prime \prime}, \theta_{1}^{\prime \prime}$ and $\theta_{2}^{\prime \prime}$ the images of $\eta_{1}^{\prime}, \theta_{1}^{\prime}$ and $\theta_{2}^{\prime}$ in $r:=E(p \otimes q, T) \in \mathcal{C}$, then $\gamma_{1}$ and $\eta_{2}^{\prime \prime}$ belong to the same block in $r$ and so do $\theta_{1}^{\prime}$ and $\theta_{2}^{\prime}$. Because $\left(\gamma_{1}, \gamma_{2}, \eta_{1}^{\prime}, \theta_{i}^{\prime}, \eta_{2}^{\prime}, \theta_{\neg i}^{\prime}\right)$ is ordered in $p \otimes q$ for some $i, \neg i \in\{1,2\}$ with $\{i, \neg i\}=\{1,2\}$, the tuple $\left(\gamma_{1}, \theta_{i}^{\prime \prime}, \eta_{2}^{\prime \prime}, \theta_{\neg i}^{\prime \prime}\right)$ is then ordered in $r$. Thus, the blocks of $\gamma_{1}$ and $\eta_{2}^{\prime \prime}$ and of $\theta_{1}^{\prime \prime}$ and $\theta_{2}^{\prime \prime}$ cross in $r$.

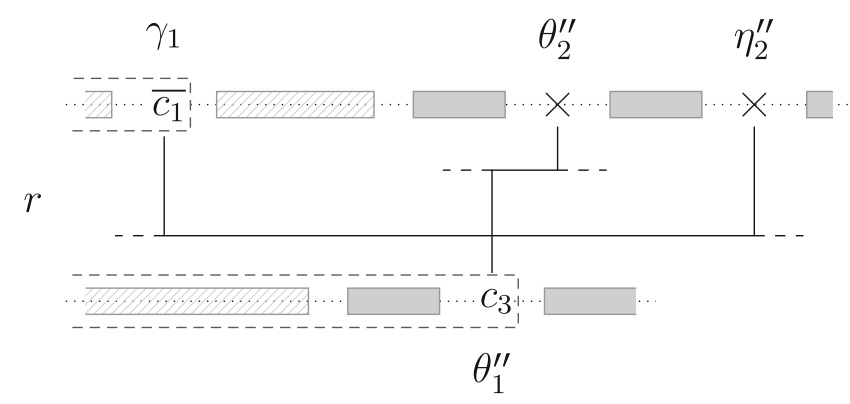

Consequently, from $\delta_{r}\left(\gamma_{1}, \theta_{1}^{\prime \prime}\right) \in X_{c_{1}, c_{3}}(\mathcal{C})$ and from

$$
\begin{aligned}
\delta_{r}\left(\gamma_{1}, \theta_{1}^{\prime \prime}\right) & =\delta_{p \otimes q}\left(\gamma_{1}, \theta_{1}^{\prime}\right)-\sigma_{p \otimes q}(T) \\
& =\delta_{p \otimes q}\left(\gamma_{1}, \theta_{1}^{\prime}\right) \\
& =\delta_{p \otimes q}\left(\gamma_{1}, \gamma_{2}\right)+\delta_{p \otimes q}\left(\gamma_{2}, \eta_{1}^{\prime}\right)+\delta_{p \otimes q}\left(\eta_{1}^{\prime}, \theta_{1}^{\prime}\right) \\
& =\delta_{p \otimes q}\left(\gamma_{1}, \gamma_{2}\right)+\delta_{p \otimes q}\left(\eta_{1}^{\prime}, \theta_{1}^{\prime}\right) \\
& =\delta_{p}\left(\gamma_{1}, \gamma_{2}\right)+\delta_{q}\left(\eta_{1}, \theta_{1}\right)
\end{aligned}
$$

it follows $\delta_{p}\left(\gamma_{1}, \gamma_{2}\right)+\delta_{q}\left(\eta_{1}, \theta_{1}\right) \in X_{c_{1}, c_{3}}(\mathcal{C})=\xi_{c_{1}, c_{3}}$. And that is what we needed to see. 
Finally, we can give the final result of this section.

Proposition 7.23 Let $\mathcal{C} \subseteq \mathcal{P}^{\circ \bullet}$ be a non-hyperoctahedral category. Then,

$$
L(\mathcal{C})=K_{\circ \circ}(\mathcal{C})=K_{\bullet \bullet}(\mathcal{C}), \quad K(\mathcal{C})=K_{\circ \bullet}(\mathcal{C})=K_{\bullet \circ}(\mathcal{C})
$$

and

$$
X(\mathcal{C})=X_{\circ \circ}(\mathcal{C})=X_{\bullet \bullet}(\mathcal{C})=X_{\circ \bullet}(\mathcal{C})=X_{\bullet \circ}(\mathcal{C})
$$

and there exist $u \in\{0\} \cup \mathbb{N}, m \in \mathbb{N}, D \subseteq\{0\} \cup \llbracket\left\lfloor\frac{m}{2}\right\rfloor \rrbracket$ and $E \subseteq\{0\} \cup \mathbb{N}$ such that the tuple $(\Sigma, L, K, X)(\mathcal{C})$ is one of the following:

\begin{tabular}{cccc}
$\Sigma(\mathcal{C})$ & $L(\mathcal{C})$ & $K(\mathcal{C})$ & $X(\mathcal{C})$ \\
\hline$u m \mathbb{Z}$ & $m \mathbb{Z}$ & $m \mathbb{Z}$ & $\mathbb{Z} \backslash D_{m}$ \\
$2 u m \mathbb{Z}$ & $m+2 m \mathbb{Z}$ & $2 m \mathbb{Z}$ & $\mathbb{Z} \backslash D_{m}$ \\
$u m \mathbb{Z}$ & $\varnothing$ & $m \mathbb{Z}$ & $\mathbb{Z} \backslash D_{m}$ \\
$\{0\}$ & $\{0\}$ & $\{0\}$ & $\mathbb{Z} \backslash E_{0}$ \\
$\{0\}$ & $\varnothing$ & $\{0\}$ & $\mathbb{Z} \backslash E_{0}$
\end{tabular}

Proof Follows from Lemmata 7.16-7.22 and the Arithmetic Lemma 7.13.

\section{Step 5: Special Relations between $\Sigma, L, K$ and $X$ depending on $F$ and $V$}

Our objective remains proving $Z(\mathcal{C}) \in Q$ for any non-hyperoctahedral category $\mathcal{C} \subseteq \mathcal{P}^{\circ}$. After studying components $F$ (Section 4 ) and $\Sigma$ (Section 6) in isolation and after investigating the images of the mappings $(F, V, L)$ (Section 5) and $(\Sigma, L, K, X)$ (Section 7), we have arrived at the point where we must take all six components of $Z=(F, V, \Sigma, L, K, X)$ into account simultaneously. Fortunately, we can capitalize on the results of Sections 4-7 in this endeavor. In consequence, it largely suffices to understand better the behavior of ( $\Sigma, L, K, X)$ as dependent on $(F, V)$ or, roughly, on $F$.

Recall from [5, Definition 4.1] that a category is non-hyperoctahedral if and only if it is case $\mathcal{O}, \mathcal{B}$ or $\mathcal{S}$ and that these cases are mutually exclusive.

\subsection{Special Relations in Case $\mathcal{S}$}

For case $\mathcal{S}$ categories $\mathcal{C} \subseteq \mathcal{P}^{\circ}$, i.e., by Proposition 4.3 assuming $F(\mathcal{C})=\mathbb{N}$, there is just a single fact about $(\Sigma, L, K, X)(\mathcal{C})$ we have to note, one about $L(\mathcal{C})$.

Proposition 8.1 $0 \in L(\mathcal{C})$ for every case $\mathcal{S}$ category $\mathcal{C} \subseteq \mathcal{P}^{\circ}$

Proof As $\hat{\jmath} \otimes \hat{\bullet} \in \mathcal{C}$, we can, by Lemma 4.1 (c), disconnect the black points in $\_\_. \in \mathcal{C}$ and obtain $\hat{\imath} \uparrow \in \mathcal{C}$. It follows $\{0\}=L(\{\{\hat{\imath} \uparrow\}) \subseteq L(\mathcal{C})$.

\subsection{Special Relations in Case $\mathcal{O}$}

For case $\mathcal{O}$ categories $\mathcal{C} \subseteq \mathcal{P}^{\circ}$, i.e., assuming $F(\mathcal{C})=\{2\}$, more than what Proposition 7.23 is able to discern can be said about $\Sigma(\mathcal{C})$ and $X(\mathcal{C})$. 


\subsubsection{Relation of $\boldsymbol{\Sigma}$ to $L$ and $K$ in Case $\mathcal{O}$}

First, we treat the total color sums of case $\mathcal{O}$ categories.

Proposition 8.2 Let $\mathcal{C} \subseteq \mathcal{P}^{\circ \bullet}$ be a case $\mathcal{O}$ category and let $m \in \mathbb{N}$.

(a) If $(L, K)(\mathcal{C})=(\varnothing, m \mathbb{Z})$, then $\Sigma(\mathcal{C})=\{0\}$.

(b) If $(L, K)(\mathcal{C})=(m \mathbb{Z}, m \mathbb{Z})$ or $(L, K)(\mathcal{C})=(m+2 m \mathbb{Z}, 2 m \mathbb{Z})$, then

$$
\Sigma(\mathcal{C})=2 u m \mathbb{Z}
$$

for some $u \in\{0\} \cup \mathbb{N}$.

Proof (a) By Proposition 7.23 there exists $\tilde{u} \in\{0\} \cup \mathbb{N}$ such that $\Sigma(\mathcal{C})=\tilde{u} m \mathbb{Z}$. We suppose $\tilde{u} \neq 0$ and derive a contradiction. As $\mathcal{C}$ is closed under erasing turns and as erasing turns does not affect total color sum, we find $p \in \mathcal{C}$ with no turns such that $\Sigma(p)=\tilde{u} m$. Because $\tilde{u} m>0$, the partition $p$ has at least one block. As all blocks of $p$ are pairs by Proposition 4.3, there is a block $B$ of $p$ with (necessarily subsequent) legs $\alpha, \beta \in B$ and $\alpha \neq \beta$. Since $p$ has no turns, all points of $p$ have normalized color $\circ$. In particular, $\alpha$ and $\beta$ do. That proves $L(\mathcal{C}) \neq \varnothing$, contradicting the assumption.

(b) Proposition 7.23 guarantees that $\Sigma(\mathcal{C})=\tilde{u} m \mathbb{Z}$ for some $\tilde{u} \in\{0\} \cup \mathbb{N}$ and that $\tilde{u}$ is even if $(L, K)(\mathcal{C})=(m+2 m \mathbb{Z}, 2 m \mathbb{Z})$. We want to show that $\tilde{u}$ is even also if $(L, K)(\mathcal{C})=(m \mathbb{Z}, m \mathbb{Z})$. If $\tilde{u}=0$, this claim is true. Hence, suppose $\tilde{u}>0$. As in Part (a), we utilize $p \in \mathcal{C}$ with no turns such that $\Sigma(p)=\tilde{u} m>0$ and, this time, also with no upper points.

For every $i \in \mathbb{N}$ with $i \leq m$ consider the set

$$
S_{i}=\left\{. j \mid j \in\left(i+m \mathbb{N}_{0}\right), j \leq \tilde{u} m\right\}
$$

comprising the $i$-th lower point and all its $m$-th neighbors to the right. Then, $\bigcup_{i=1}^{m} S_{i}$ comprises all points of $p$ and $\left|S_{i}\right|=\tilde{u}$ for every $i \in \mathbb{N}$ with $i \leq m$.

The sets $S_{1}, \ldots, S_{m}$ must all be subpartitions of $p$ : Otherwise, we find $j, j^{\prime} \in \mathbb{N}$ with $j<j^{\prime} \leq \tilde{u} m$ and $j^{\prime}-j \notin m \mathbb{Z}$ such that.$j$ and.$j^{\prime}$ belong to the same block. As all of ]. $\left.j, j^{\prime}\right]_{p}$ has normalized color $\circ$,

$$
\left.\left.\left.\left.\delta_{p}\left(\bullet j, j^{\prime}\right)=\sigma_{p}(]_{\bullet} j, j^{\prime}\right]_{p}\right)=\mid\right]_{\bullet} j, j^{\prime}\right]_{p} \mid=j^{\prime}-j \notin m \mathbb{Z} .
$$

That contradicts the assumption $L(\mathcal{C})=m \mathbb{Z}$.

Because all blocks of $p$ are pairs by Proposition 4.3, subpartitions of $p$ have even cardinality. We conclude $\tilde{u}=\left|S_{1}\right| \in 2 \mathbb{Z}$, which then proves the claim.

\subsubsection{Relation of $X$ to $L$ and $K$ in Case $\mathcal{O}$}

When studying $X(\mathcal{C})$ further for case $\mathcal{O}$ categories $\mathcal{C} \subseteq \mathcal{P}^{\bullet \bullet}$, it is best to distinguish whether $(L \cup K)(\mathcal{C})$ contains non-zero elements or not.

Proposition 8.3 Let $\mathcal{C} \subseteq \mathcal{P}^{\circ}$ be a case $\mathcal{O}$ category and let $m \in \mathbb{N}$.

(a) If $(L, K)(\mathcal{C})=(m+2 m \mathbb{Z}, 2 m \mathbb{Z})$, then $X(\mathcal{C})=\mathbb{Z}$ or $X(\mathcal{C})=\mathbb{Z} \backslash m \mathbb{Z}$.

(b) If $(L, K)(\mathcal{C})=(m \mathbb{Z}, m \mathbb{Z})$ or $(L, K)(\mathcal{C})=(\varnothing, m \mathbb{Z})$, then $X(\mathcal{C})=\mathbb{Z}$.

Proof No matter which of the three values $(L, K)(\mathcal{C})$ takes, by Proposition 7.23 the set $X(\mathcal{C})$ is $m$-periodic. Therefore, showing $\llbracket m \rrbracket \subseteq X(\mathcal{C})$ already implies $X(\mathcal{C})=\mathbb{Z}$. Likewise, provided $m \geq 2$, establishing $\llbracket m-1 \rrbracket \subseteq X(\mathcal{C})$ forces the conclusion that $X(\mathcal{C})=\mathbb{Z} \backslash m \mathbb{Z}$ or $X(\mathcal{C})=\mathbb{Z}$. 
(a) First, let $(L, K)(\mathcal{C})=(m+2 m \mathbb{Z}, 2 m \mathbb{Z})$. If $m=1$, the 1-periodicity of $X(\mathcal{C})$ immediately implies $X(\mathcal{C})=\varnothing$ or $X(\mathcal{C})=\mathbb{Z}$. Hence, we can suppose $m \geq 2$ and only need to prove $\llbracket m-1 \rrbracket \subseteq X(\mathcal{C})$ by the initial remark.

Proposition 7.23 lets us infer $K_{\circ \circ}(\mathcal{C})=m+2 m \mathbb{Z}$. Hence, we find a partition $p \in$ $\mathcal{C} \subseteq \mathcal{P}_{2}^{\circ \bullet}$, therein a block $\{\alpha, \beta\}$ with $\alpha$ and $\beta$ both of normalized color $\circ$, with $\alpha \neq \beta$ and with $\delta_{p}(\alpha, \beta)=m$. Without infringing on any of these assumptions we can additionally suppose that there are no turns $T$ in $p$ such that $T \subseteq] \alpha, \beta\left[{ }_{p}\right.$ (otherwise we erase them). Then, all of $] \alpha, \beta[p$ has the same normalized color $c \in\{\circ, \bullet\}$.

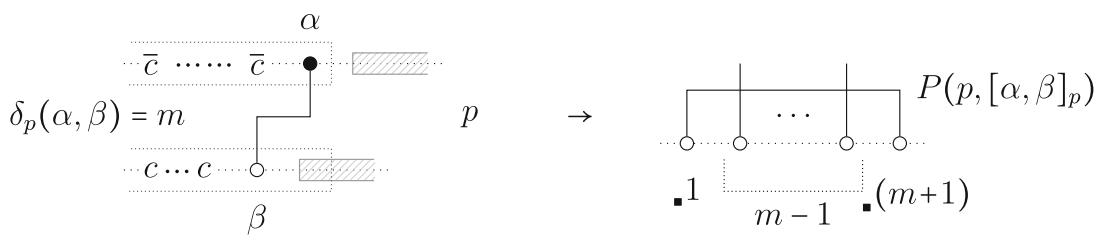

Because $\alpha$ and $\beta$ also identically have normalized color $\circ$,

$$
\left.\left.m=\delta_{p}(\alpha, \beta)=\sigma_{p}(] \alpha, \beta\right]_{p}\right)= \begin{cases}\mid] \alpha, \beta]_{p} \mid & \text { if } c=\circ \\ -\mid] \alpha, \beta]_{p} \mid & \text { otherwise }\end{cases}
$$

As $m>0$, the only option is $c=0$. That means $[\alpha, \beta]_{p}$ consists of $m+1$ points of normalized color $\circ$.

By definition of the projection operation and by Lemma 3.3, it is possible to further add the premise $p=P\left(p,[\alpha, \beta]_{p}\right)$ without impacting any of the previous assumptions. Now, $p$ is also projective and $[\alpha, \beta]_{p}=[.1,(m+1)]_{p}$ is its lower row.

For every $j \in \mathbb{N}$ with $1<j<m+1$ the point.$j$ belongs to a through block: Assuming otherwise, forces us to accept the existence of $j, j^{\prime} \in \mathbb{N}$ with $1<j<j^{\prime}<m+1$ such that $j$ and.$j^{\prime}$ belong to the same block. But then, the uniform color $\circ$ of $[\alpha, \beta]_{p}$ implies

$$
1 \leq \delta_{p}\left(\bullet j, j^{\prime}\right)=j^{\prime}-j \leq m-2 \leq m-1
$$

and thus $L(\mathcal{C}) \cap\{1, \ldots, m-1\} \neq \varnothing$, contradicting $L(\mathcal{C}) \subseteq m \mathbb{Z}$.

Thus we have shown that $\alpha=.1$ and.$j$ belong to crossing blocks for every $j \in \mathbb{N}$ with $1<j<m+1$. Because $\delta_{p}(\alpha, j)=j-1$ for every such $j$, this proves $\llbracket m-1 \rrbracket \subseteq X(\mathcal{C})$. And that is what we needed to show.

(b) Let $(L, K)(\mathcal{C})$ be given by $(m \mathbb{Z}, m \mathbb{Z})$ or $(\varnothing, m \mathbb{Z})$. We adapt the proof of Part (a). However, this time, we do not yet impose any restriction on $m$.

Proposition 7.23 assures us that $K_{\circ}(\mathcal{C})=K(\mathcal{C})=m \mathbb{Z}$. Hence, we again find $p \in \mathcal{C}$, a block $B$ of $p$ and legs $\alpha, \beta \in B$ with $\alpha \neq \beta$, with $] \alpha, \beta\left[{ }_{p} \cap B=\varnothing\right.$ and with $\delta_{p}(\alpha, \beta)=m$, but this time, such that $\alpha$ is of normalized color $\circ$ and $\beta$ of normalized color $\bullet$. By the same argument as before we can assume that all points of $] \alpha, \beta[p$ share the same normalized color. Then, the deviating assumption on the colors of $\alpha$ and $\beta$ implies $\left.m=\delta_{p}(\alpha, \beta)=\sigma_{p}(] \alpha, \beta\left[_{p}\right)=\mid\right] \alpha, \beta\left[{ }_{p} \mid\right.$, which forces $[\alpha, \beta]_{p}$ to consist of exactly $m+2$ points (rather than $m+1$ as in Part (a)), the first $m+1$ of which have normalized color $\circ$. Once more, we can assume $p=P\left(p,[\alpha, \beta]_{p}\right)$. 


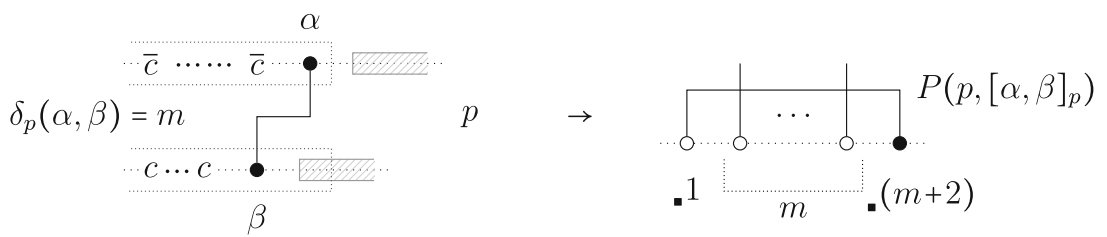

If $m=1$, then $F(\{p\})=\{2\}$ requires the unique point $.2 \in] .1, .3[p$ to belong to a through block, proving $1 \in X(\mathcal{C})$ and thus $X(\mathcal{C})=\mathbb{Z}$ as claimed. Hence, suppose $m \geq 2$ in the following.

We prove that only through blocks intersect $]_{\bullet} 1,(m+2)\left[{ }_{p}\right.$ : Supposing that,$j$ and - $j^{\prime}$, where $j, j^{\prime} \in \mathbb{N}$ and $1<j<j^{\prime}<m+2$, belong to the same block requires us to believe, as both.$j$ and.$j^{\prime}$ are $\circ$-colored, that

$$
1 \leq \delta_{p}\left(\mathbf{\bullet} j, j^{\prime}\right)=j^{\prime}-j \leq(m+1)-2=m-1
$$

and thus $L(\mathcal{C}) \cap\{1, \ldots, m-1\} \neq \varnothing$. As this would contradict the assumption $L(\mathcal{C}) \subseteq m \mathbb{Z}$, this cannot be the case.

Now, the conclusion that the blocks of .1 and of.$j$ cross for every $j \in \mathbb{N}$ with $1<j<m+2$ and the fact $\delta_{p}(.1, \ldots j)=j-1$ let us deduce $\llbracket m \rrbracket \subseteq X(\mathcal{C})$, which is what needed to see.

Proposition 8.4 Let $\mathcal{C} \subseteq \mathcal{P}^{\circ}$ be a case $\mathcal{O}$ category.

(a) If $(L, K)(\mathcal{C})=(\{0\},\{0\})$, then $X(\mathcal{C})=\mathbb{Z} \backslash N_{0}$ for a subsemigroup $N$ of $(\mathbb{N},+)$.

(b) If $(L, K)(\mathcal{C})=(\varnothing,\{0\})$, then there exists a subsemigroup $N$ of $(\mathbb{N},+)$ such that $X(\mathcal{C})=\mathbb{Z} \backslash N_{0}$ or $X(\mathcal{C})=\mathbb{Z} \backslash N_{0}^{\prime}$.

Proof Let $(L, K)(\mathcal{C})$ be given by $(\{0\},\{0\})$ or $(\varnothing,\{0\})$. We show the two claims jointly in two steps:

Step 1: First, we prove that there exists a subsemigroup $N$ of $(\mathbb{N},+)$ such that $X(\mathcal{C})=$ $\mathbb{Z} \backslash N_{0}$ or $X(\mathcal{C})=\mathbb{Z} \backslash N_{0}^{\prime}$. That in itself requires two steps as well.

Step 1.1: Recall from [3, Definition 4.1] that by $\mathcal{S}_{0}$ we denote the set of all $p \in \mathcal{P}_{2}^{\circ \bullet}$ with $\sigma_{p}(B)=0$ and $\delta_{p}(\alpha, \beta)=0$ for all blocks $B$ of $p$ and all $\alpha, \beta \in B$. We justify that it suffices to prove

$$
\{|z| \mid z \in X(\mathcal{C})\} \backslash\{0\} \stackrel{!}{\subseteq}\left\{|z| \mid z \in X\left(\mathcal{C} \cap \mathcal{S}_{0}\right)\right\}
$$

in order to verify the assertion of Step 1 .

Indeed, in [4, Theorem 8.3, Lemmata 8.1 (b) and 7.16 (c)] it was shown that for every category $\mathcal{I} \subseteq \mathcal{S}_{0}$ there exists a subsemigroup $N$ of $(\mathbb{N},+)$ such that

$$
\{|z| \mid z \in X(\mathcal{I})\} \backslash\{0\}=\mathbb{N} \backslash N .
$$

The set $\mathcal{S}_{0}$ is a category by [3, Proposition 5.3], which means that so is $\mathcal{C} \cap \mathcal{S}_{0}$. Thus, we find a corresponding subsemigroup $N$ for the special case $\mathcal{I}=\mathcal{C} \cap \mathcal{S}_{0}$. If we now suppose (*), which can immediately be sharpened to

$$
\{|z| \mid z \in X(\mathcal{C})\} \backslash\{0\}=\left\{|z| \mid z \in X\left(\mathcal{C} \cap \mathcal{S}_{0}\right)\right\} \backslash\{0\},
$$

that implies

$$
\{|z| \mid z \in X(\mathcal{C})\} \backslash\{0\}=\mathbb{N} \backslash N .
$$


As we know $X(\mathcal{C})=-X(\mathcal{C})$ by Proposition 7.23, this is equivalent to

$$
X(\mathcal{C}) \backslash\{0\}=\mathbb{Z} \backslash N_{0}^{\prime}
$$

and thus the claim of Step 1. Hence, it is indeed sufficient to show $(*)$.

Step 1.2: We prove $(*)$. As $\mathcal{C} \subseteq \mathcal{P}_{2}^{\circ \bullet}$ by Proposition 4.3, we are assured by Lemma 7.15 and Proposition 7.23 that $X(\mathcal{C})=X_{c_{1}, c_{2}}\left(\mathcal{C} \cap \mathcal{P}_{2}^{\circ}\right)$ for all $c_{1}, c_{2} \in\{\circ, \bullet\}$. Now, let $z \in X(\mathcal{C}) \backslash\{0\}$ be arbitrary. By definition we find $p \in \mathcal{C} \cap \mathcal{P}_{2}^{\circ \bullet}$ and therein crossing blocks $B_{1}$ and $B_{2}$ as well as points $\alpha_{1} \in B_{1}$ and $\alpha_{2} \in B_{2}$ such that $\delta_{p}\left(\alpha_{1}, \alpha_{2}\right)=z$. Then, there exist points $\beta_{1} \in B_{1}$ and $\beta_{2} \in B_{2}$ such that $\alpha_{1} \neq \beta_{1}$ and $\alpha_{2} \neq \beta_{2}$ and such that either $\left(\alpha_{1}, \alpha_{2}, \beta_{1}, \beta_{2}\right)$ or $\left(\alpha_{2}, \alpha_{1}, \beta_{2}, \beta_{1}\right)$ is ordered in $p$. As $\Sigma(\mathcal{C})=\{0\}$ by Proposition 7.23 and thus $\Sigma(p)=$ 0 , we know $\delta_{p}\left(\alpha_{2}, \alpha_{1}\right)=-\delta_{p}\left(\alpha_{1}, \alpha_{2}\right)$ by [5, Lemma 2.1]. Hence, by renaming $B_{1}$ and $B_{2}$ if necessary we can, at the cost of weakening $\delta_{p}\left(\alpha_{1}, \alpha_{2}\right)=z$ to $\left|\delta_{p}\left(\alpha_{1}, \alpha_{2}\right)\right|=|z|$, assume that $\left(\alpha_{1}, \alpha_{2}, \beta_{1}, \beta_{2}\right)$ is ordered. As $\mathcal{C} \cap \mathcal{P}_{2}^{\circ \bullet}$ is closed under erasing turns and as $\left.\left(B_{1} \cup B_{2}\right) \cap\right] \alpha_{1}, \alpha_{2}[p=\varnothing$ we can further suppose that no turns $T$ exist in $p$ with $T \subseteq] \alpha_{1}, \alpha_{2}[p$. In other words, there is $c \in\{\circ, \bullet\}$ such that every point in $] \alpha_{1}, \alpha_{2}[p$ has normalized color $c$.

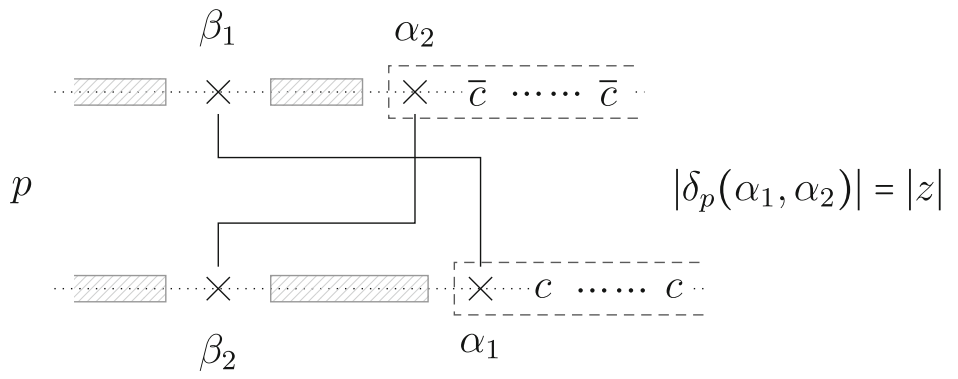

Even further, by Lemma 3.3 none of the previous assumptions are violated by assuming that $p=P\left(p,\left[\alpha_{1}, \beta_{1}\right]_{p}\right)$. Then, $\beta_{2}$ is the counterpart of $\alpha_{2}$ on the upper row, $\alpha_{1} \in\left[\beta_{2}, \alpha_{2}\right]_{p}$ and $\beta_{1} \notin\left[\beta_{2}, \alpha_{2}\right]_{p}$. If we let $\epsilon$ be the predecessor of $\alpha_{1}$, i.e., if $\epsilon$ is the leftmost upper point of $p$, then $\left(\beta_{2}, \epsilon, \alpha_{1}, \alpha_{2}, \beta_{1}\right)$ is ordered.

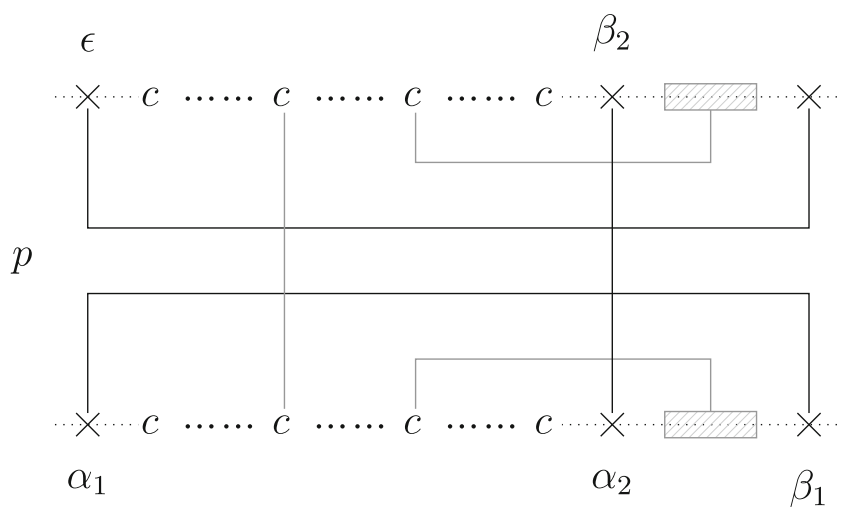

Recall that there are no turns $T$ in $p$ with $T \subseteq] \alpha_{1}, \alpha_{2}\left[p\right.$. As $p=p^{*}$, there are none with $T \subseteq] \beta_{2}, \epsilon[p$ either. That means every point in $] \alpha_{1}, \alpha_{2}[p$ has normalized color $c$ and every point in $] \beta_{2}, \epsilon[p$ normalized color $\bar{c}$. We can also say a lot about the blocks of $p$ which intersect $\left[\beta_{2}, \alpha_{2}\right]_{p}$ : If a point $\left.\theta_{1} \in\right] \alpha_{1}, \alpha_{2}[p$ belongs to a through block it must be connected to its counterpart on the upper row because $p \in \mathcal{P}_{2}^{\circ \bullet}$ is projective. If $\theta_{1}$ belongs to a non-through block instead, then the partner $\theta_{2}$ of $\theta_{1}$ must lie outside $\left[\beta_{2}, \alpha_{2}\right]_{p}$ : Supposing 
otherwise, i.e., $\left.\theta_{2} \in\right] \alpha_{1}, \alpha_{2}\left[p\right.$, produces a contradiction: If $\left(\alpha_{1}, \theta_{i}, \theta_{\neg i}, \beta_{2}\right)$ with $i, \neg i \in\{1,2\}$ and $\{i, \neg i\}=\{1,2\}$ is ordered, then, as all points in $\left[\theta_{i}, \theta_{\neg i}\right]_{p}$ are $c$-colored, the consequence $\left.\left.\left|\delta_{p}\left(\theta_{i}, \theta_{\neg i}\right)\right|=\mid\right] \theta_{i}, \theta_{\neg i}\right]_{p} \mid>0$ violates $L(\mathcal{C}) \subseteq\{0\}$, which follows from $K(\mathcal{C})=\{0\}$ by Proposition 7.23.

Define $p^{\prime}:=P\left(p,\left[\beta_{2}, \alpha_{2}\right]_{p}\right) \in \mathcal{C} \cap \mathcal{P}_{2}^{\circ \bullet}$ and denote by $\beta_{2}^{\prime}, \epsilon^{\prime}, \alpha_{1}^{\prime}$ and $\alpha_{2}^{\prime}$ the images in $p^{\prime}$ of $\beta_{2}, \epsilon, \alpha_{1}$ and $\alpha_{2}$, respectively. In $p^{\prime}$ the leftmost lower point $\beta_{2}^{\prime}$ and the rightmost lower point $\alpha_{2}^{\prime}$ form a block. The points $\epsilon^{\prime}, \alpha_{1}^{\prime} \in\left[\beta_{2}^{\prime}, \alpha_{2}^{\prime}\right]$ are each paired with their respective counterpart on the upper row. In particular the blocks of $\alpha_{1}^{\prime}$ and $\alpha_{2}^{\prime}$ cross in $p^{\prime}$.

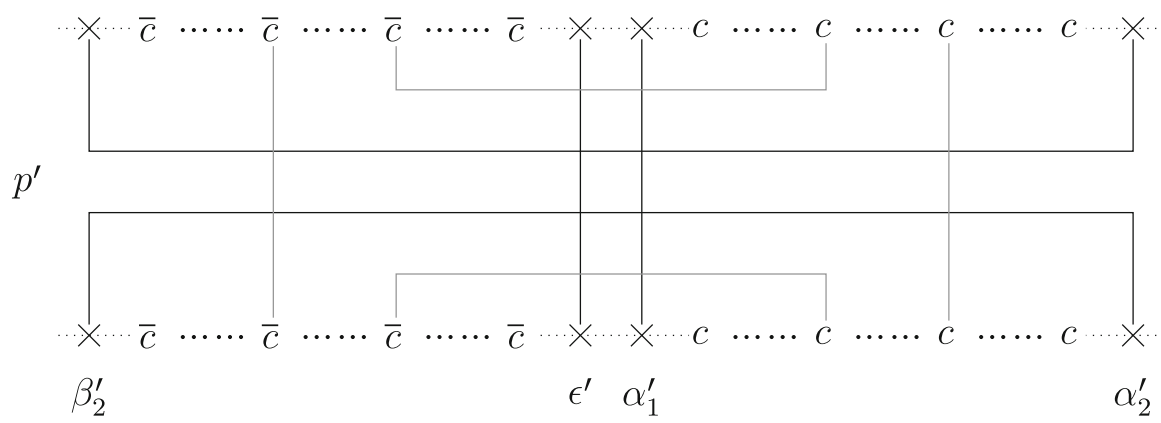

Our knowledge about the blocks of $p$ intersecting $\left[\beta_{2}, \alpha_{2}\right]_{p}$ lets us draw the following conclusions about the blocks of $p^{\prime}$ : A point in $] \alpha_{1}^{\prime}, \alpha_{2}^{\prime}[p$ is either partnered with its reflection at the center $\left[\epsilon^{\prime}, \alpha_{1}^{\prime}\right]_{p^{\prime}}$ of the lower row of $p^{\prime}$ in $] \beta_{2}^{\prime}, \epsilon^{\prime}\left[p^{\prime}\right.$ or, as $p^{\prime}$ is projective, it is partnered with its counterpart on the opposite row. As $] \beta_{2}^{\prime}, \epsilon^{\prime}\left[p_{p^{\prime}}\right.$ is uniformly $\bar{c}$-colored and $] \alpha_{1}^{\prime}, \alpha_{2}^{\prime}\left[_{p^{\prime}}\right.$ uniformly $c$-colored, that means that all blocks emanating from $] \beta_{2}^{\prime}, \epsilon^{\prime}\left[p^{\prime} \cup\right] \alpha_{1}^{\prime}, \alpha_{2}^{\prime}[p$ are neutral. But then, all blocks of $p^{\prime}$ are neutral. Due to $L(\mathcal{C}) \subseteq\{0\}$ and $K(\mathcal{C})=\{0\}$, this is already enough to know $p^{\prime} \in \mathcal{S}_{0}$. Because $\delta_{p^{\prime}}\left(\alpha_{1}^{\prime}, \alpha_{2}^{\prime}\right)=\delta_{p}\left(\alpha_{1}, \alpha_{2}\right)$, that proves $|z|=$ $\left|\delta_{p}\left(\alpha_{1}, \alpha_{2}\right)\right|=\left|\delta_{p^{\prime}}\left(\alpha_{1}^{\prime}, \alpha_{2}\right)\right| \in\left\{|z| \mid z \in X\left(\mathcal{C} \cap \mathcal{S}_{0}\right)\right\}$. As $z$ was arbitrary, $(*)$ holds true and Part (b) has been proven.

Step 2: In order to prove Part (a) it remains to show $0 \in X(\mathcal{C})$ provided $L(\mathcal{C})=\{0\}$. Under this latter assumption, by Proposition 7.23 we infer $K_{\circ \circ}(\mathcal{C})=\{0\}$. Hence, we find $p \in \mathcal{C}$, therein a block $B$ and legs $\alpha, \beta \in B$ of normalized color $\circ$ with $\alpha \neq \beta$, with $] \alpha, \beta\left[{ }_{p} \cap B=\varnothing\right.$ and with $\delta_{p}(\alpha, \beta)=0$. As in the proof of Proposition 8.3 we can assume that there are no turns $T$ in $p$ such that $T \subseteq] \alpha, \beta[p$, i.e. that all points in $] \alpha, \beta{ }_{p}$ have the same normalized color $c \in\{\circ, \bullet\}$. From

$$
\left.\left.0=\delta_{p}(\alpha, \beta)=\sigma_{p}(] \alpha, \beta\right]_{p}\right)=\sigma_{p}(] \alpha, \beta[p)+\sigma_{p}(\{\beta\})= \begin{cases}\mid] \alpha, \beta\left[{ }_{p} \mid+1\right. & \text { if } c=0, \\ -\mid] \alpha, \beta\left[{ }_{p} \mid+1\right. & \text { otherwise }\end{cases}
$$

and from $\mid] \alpha, \beta\left[{ }_{p} \mid \geq 0\right.$ it follows that $c=\bullet$ and that $] \alpha, \beta\left[{ }_{p}\right.$ is a singleton set. Emulating the proof of Proposition 8.3 further, we can assume $p=P\left(p,[\alpha, \beta]_{p}\right)$.

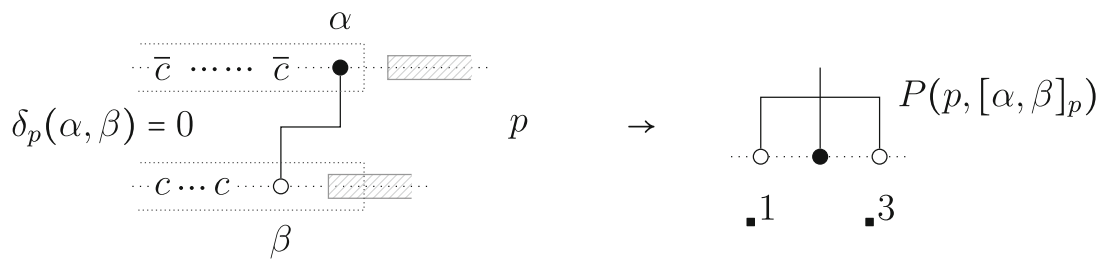


Then, the lower row $[\alpha, \beta]_{p}=[.1, .3]_{p}$ of $p$ has coloration $\circ \bullet \circ$. As $p \in \mathcal{P}_{2}^{\circ \bullet}$ and as $p$ is projective, the block of .2 is the pair $\{2,+2\}$. That means the blocks of $\alpha=1$ and .2 cross, implying $0=\delta_{p}\left(\_1, .2\right) \in X(\mathcal{C})$. That concludes the proof.

\section{Step 6: Synthesis}

Combining the results from Sects. 4-8, we are able to show the main theorem.

Theorem 9.1 $Z(\mathcal{C}) \in Q$ for every non-hyperoctahedral category $\mathcal{C} \subseteq \mathcal{P}^{\circ \bullet}$

Proof By Lemma 7.23 there exist $u \in\{0\} \cup \mathbb{N}, m \in \mathbb{N}, D \subseteq\{0\} \cup \llbracket\left\lfloor\frac{m}{2}\right\rfloor \rrbracket$ and $E \subseteq\{0\} \cup \mathbb{N}$ such that the tuple $(\Sigma, L, K, X)(\mathcal{C})$ is given by one of the following:

\begin{tabular}{cccc}
$\Sigma$ & $L$ & $K$ & $X$ \\
\hline$u m \mathbb{Z}$ & $m \mathbb{Z}$ & $m \mathbb{Z}$ & $\mathbb{Z} \backslash D_{m}$ \\
$2 u m \mathbb{Z}$ & $m+2 m \mathbb{Z}$ & $2 m \mathbb{Z}$ & $\mathbb{Z} \backslash D_{m}$ \\
$u m \mathbb{Z}$ & $\varnothing$ & $m \mathbb{Z}$ & $\mathbb{Z} \backslash D_{m}$ \\
$\{0\}$ & $\{0\}$ & $\{0\}$ & $\mathbb{Z} \backslash E_{0}$ \\
$\{0\}$ & $\varnothing$ & $\{0\}$ & $\mathbb{Z} \backslash E_{0}$
\end{tabular}

We treat the three cases $\mathcal{O}, \mathcal{B}$ and $\mathcal{S}$ individually. The formulaic presentation will mirror that of Definition 2.5 exactly, to facilitate cross-checking.

Case $\mathcal{B}$ : First, let $\mathcal{C}$ be case $\mathcal{B}$. Proposition 4.3 (c) implies $F(\mathcal{C})=\{1,2\}$. So, we can immediately add the column for $F(\mathcal{C})$ to table $(*)$. Further, Proposition 5.1 (c) shows $V(\mathcal{C})=$ $\pm\{0,1,2\}$ if and only if $L(\mathcal{C}) \neq \varnothing$ and $V(\mathcal{C})= \pm\{0,1\}$ otherwise. That allows us to fill in the column for $V(\mathcal{C})$ as well. The result is that $Z(\mathcal{C})$ concurs with a row of the table

\begin{tabular}{cccccc}
$F$ & $V$ & $\Sigma$ & $L$ & $K$ & $X$ \\
\hline$\{1,2\}$ & $\pm\{0,1,2\}$ & $u m \mathbb{Z}$ & $m \mathbb{Z}$ & $m \mathbb{Z}$ & $\mathbb{Z} \backslash D_{m}$ \\
$\{1,2\}$ & $\pm\{0,1,2\}$ & $2 u m \mathbb{Z}$ & $m+2 m \mathbb{Z}$ & $2 m \mathbb{Z}$ & $\mathbb{Z} \backslash D_{m}$ \\
$\{1,2\}$ & $\pm\{0,1\}$ & $u m \mathbb{Z}$ & $\varnothing$ & $m \mathbb{Z}$ & $\mathbb{Z} \backslash D_{m}$ \\
$\{1,2\}$ & $\pm\{0,1,2\}$ & $\{0\}$ & $\{0\}$ & $\{0\}$ & $\mathbb{Z} \backslash E_{0}$ \\
$\{1,2\}$ & $\pm\{0,1\}$ & $\{0\}$ & $\varnothing$ & $\{0\}$ & $\mathbb{Z} \backslash E_{0}$
\end{tabular}

for some $u \in\{0\} \cup \mathbb{N}, m \in \mathbb{N}, D \subseteq\{0\} \cup \llbracket\left\lfloor\frac{m}{2}\right\rfloor \rrbracket$ and $E \subseteq\{0\} \cup \mathbb{N}$. Hence, by Definition 2.5, we have shown $Z(\mathcal{C}) \in Q$ if $\mathcal{C}$ is case $\mathcal{B}$.

Case $\mathcal{S}$ : Next, let $\mathcal{C}$ be case $\mathcal{S}$. Propositions $4.3(\mathrm{~d})$ and 5.1 (d) guarantee $F(\mathcal{C})=\mathbb{N}$ and $V(\mathcal{C})=\mathbb{Z}$. Hence, we can fill in the columns for $F$ and $V$ in $(*)$ once more. Moreover, $0 \in$ $L(\mathcal{C})$ by Proposition 8.1. Thus, we can exclude that $(\Sigma, L, K, X)(\mathcal{C})$ is given by the second, third or fifth rows of (*). In other words, there are $u \in\{0\} \cup \mathbb{N}, m \in \mathbb{N}, D \subseteq\{0\} \cup \llbracket\left\lfloor\frac{m}{2}\right\rfloor \rrbracket$ and $E \subseteq\{0\} \cup \mathbb{N}$ such that $Z(\mathcal{C})$ is given by one of the rows of the following table:

\begin{tabular}{cccccc}
$F$ & $V$ & $\Sigma$ & $L$ & $K$ & $X$ \\
\hline $\mathbb{N}$ & $\mathbb{Z}$ & $u m \mathbb{Z}$ & $m \mathbb{Z}$ & $m \mathbb{Z}$ & $\mathbb{Z} \backslash D_{m}$ \\
$\mathbb{N}$ & $\mathbb{Z}$ & $\{0\}$ & $\{0\}$ & $\{0\}$ & $\mathbb{Z} \backslash E_{0}$
\end{tabular}

And, by Definition 2.5, this means $Z(\mathcal{C}) \in Q$ for $\mathcal{C}$ in case $\mathcal{S}$.

Case $\mathcal{O}$ : Lastly, let $\mathcal{C}$ be case $\mathcal{O}$. Once more, Propositions 4.3 (b) and 5.1 (b) give, on the one hand, $F(\mathcal{C})=\{2\}$ and, on the other hand, $V(\mathcal{C})= \pm\{0,2\}$ if $L(\mathcal{C}) \neq \varnothing$ and $V(\mathcal{C})=\{0\}$ 
otherwise. That enables us to fill in the columns for $F(\mathcal{C})$ and $V(\mathcal{C})$ in $(*)$ :

\begin{tabular}{cccccc}
$F$ & $V$ & $\Sigma$ & $L$ & $K$ & $X$ \\
\hline$\{2\}$ & $\pm\{0,2\}$ & $u m \mathbb{Z}$ & $m \mathbb{Z}$ & $m \mathbb{Z}$ & $\mathbb{Z} \backslash D_{m}$ \\
$\{2\}$ & $\pm\{0,2\}$ & $2 u m \mathbb{Z}$ & $m+2 m \mathbb{Z}$ & $2 m \mathbb{Z}$ & $\mathbb{Z} \backslash D_{m}$ \\
$\{2\}$ & $\{0\}$ & $u m \mathbb{Z}$ & $\varnothing$ & $m \mathbb{Z}$ & $\mathbb{Z} \backslash D_{m}$ \\
$\{2\}$ & $\pm\{0,2\}$ & $\{0\}$ & $\{0\}$ & $\{0\}$ & $\mathbb{Z} \backslash E_{0}$ \\
$\{2\}$ & $\{0\}$ & $\{0\}$ & $\varnothing$ & $\{0\}$ & $\mathbb{Z} \backslash E_{0}$
\end{tabular}

This is not yet what we claim as this range is not contained in $Q$. We need to exclude certain values for $u, D$ and $E$ by taking into account the results of Section 8.2. This we shall do on a row-by-row basis.

Case $\mathcal{O} .1$ : First, suppose $(L, K)(\mathcal{C})=(m \mathbb{Z}, m \mathbb{Z})$ for some $m \in \mathbb{N}$, as in the first row of Table (**). Then $\Sigma(\mathcal{C}) \subseteq 2 m \mathbb{Z}$ (corresponding to parameters $u \in 2 \mathbb{Z}$ ) according to Proposition 8.2 (b). Moreover, $X(\mathcal{C})=\mathbb{Z}$ (corresponding to $D=\varnothing$ ) as seen in Proposition 8.3 (b). Hence, we can replace the first row of Table $(* *)$ by

$$
\begin{array}{cccccc}
F & V & \Sigma & L & K & X \\
\hline\{2\} & \pm\{0,2\} & 2 u m \mathbb{Z} & m \mathbb{Z} & m \mathbb{Z} & \mathbb{Z}
\end{array}
$$

still for parameters $u \in\{0\} \cup \mathbb{N}$ and $m \in \mathbb{N}$ exactly as before.

Case $\mathcal{O} .2$ : Now, proceeding to the second row of Table $(* *)$, let $(L, K)(\mathcal{C})=(m+$ $2 m \mathbb{Z}, 2 m \mathbb{Z}$ ) for some $m \in \mathbb{N}$. By Proposition 8.3 (a) the only two values $X(\mathcal{C})$ can possibly take are $\mathbb{Z}$ and $\mathbb{Z} \backslash m \mathbb{Z}$ (corresponding to $D=\varnothing$ and $D=\{0\}$, respectively). Thus, we can delete the second row of Table (**) and insert the two new rows

$$
\begin{array}{cccccc}
F & V & \Sigma & L & K & X \\
\hline\{2\} & \pm\{0,2\} & 2 u m \mathbb{Z} & m+2 m \mathbb{Z} & 2 m \mathbb{Z} & \mathbb{Z} \\
\{2\} & \pm\{0,2\} & 2 u m \mathbb{Z} & m+2 m \mathbb{Z} & 2 m \mathbb{Z} & \mathbb{Z} \backslash m \mathbb{Z}
\end{array}
$$

in its stead, still for parameters $m \in \mathbb{N}$ and $u \in\{0\} \cup \mathbb{N}$.

Case $\mathcal{O} .3$ : Next, assume $(L, K)(\mathcal{C})=(\varnothing, m \mathbb{Z})$ for some $m \in \mathbb{N}$ as in row three of Table $(* *)$. Then, in fact, $\Sigma(\mathcal{C})=\{0\}$ as seen in Proposition $8.2($ a). Furthermore, $X(\mathcal{C})=\mathbb{Z}$ by Proposition 8.3 (b). Hence, we rewrite the third row of $(* *)$ as

$$
\begin{array}{llllll}
F & V & \Sigma & L & K & X \\
\{2\} & \{0\} & \{0\} & \varnothing & m \mathbb{Z} & \mathbb{Z}
\end{array}
$$

depending only on the parameter $m \in \mathbb{N}$.

Case O.4: Let $(L, K)(\mathcal{C})=(\{0\},\{0\})$, i.e., consider the fourth row of Table $(* *)$. Then, $X(\mathcal{C})=\mathbb{Z} \backslash N_{0}$ for some subsemigroup of $(\mathbb{N},+)$ by Proposition 8.4 (a) (corresponding to $E=N$ being a subsemigroup). Accordingly, we can replace the fourth row of Table (**) by

$$
\begin{array}{cccccc}
F & V & \Sigma & L & K & X \\
\hline\{2\} & \pm\{0,2\} & \{0\} & \varnothing & \{0\} & \mathbb{Z} \backslash N_{0}
\end{array}
$$

for a new table parameter $N$, running through all subsemigroups of $(\mathbb{N},+)$.

Case O.5: Lastly, suppose $(L, K)(\mathcal{C})=(\varnothing,\{0\})$ as in the fifth row of Table (**). In Proposition 8.4 (a) we showed $X(\mathcal{C})$ is of the form $\mathbb{Z} \backslash N_{0}$ or $\mathbb{Z} \backslash N_{0}^{\prime}$ for some subsemigroup $N$ of $(\mathbb{N},+)$ (corresponding to $E=N$ and $E=\{0\} \cup N$, respectively). Thus, strike the last 
row of Table $(* *)$ and append the two rows

$$
\begin{array}{cccccc}
F & V & \Sigma & L & K & X \\
\hline\{2\} & \{0\} & \{0\} & \varnothing & \{0\} & \mathbb{Z} \backslash N_{0} \\
\{2\} & \{0\} & \{0\} & \varnothing & \{0\} & \mathbb{Z} \backslash N_{0}^{\prime}
\end{array}
$$

to the table, with $N$ being a subsemigroup of $(\mathbb{N},+)$.

Synthesis in case $\mathcal{O}$ : If we combine the results of Cases 1-5, then we can say that there exist $m \in \mathbb{N}, u \in\{0\} \cup \mathbb{N}$ and a subsemigroup $N$ of $(\mathbb{N},+)$ such that $Z(\mathcal{C})$ is given by one of the rows of the following table:

\begin{tabular}{cccccc}
$F$ & $V$ & $S$ & $L$ & $K$ & $X$ \\
\hline$\{2\} \pm\{0,2\}$ & $2 u m \mathbb{Z}$ & $m \mathbb{Z}$ & $m \mathbb{Z}$ & $\mathbb{Z}$ \\
$\{2\} \pm\{0,2\}$ & $2 u m \mathbb{Z}$ & $m+2 m \mathbb{Z}$ & $2 m \mathbb{Z}$ & $\mathbb{Z}$ \\
$\{2\}$ & $\pm\{0,2\}$ & $2 u m \mathbb{Z}$ & $m+2 m \mathbb{Z}$ & $2 m \mathbb{Z}$ & $\mathbb{Z} \backslash m \mathbb{Z}$ \\
$\{2\}$ & $\{0\}$ & $\{0\}$ & $\varnothing$ & $m \mathbb{Z}$ & $\mathbb{Z}$ \\
$\{2\} \pm\{0,2\}$ & $\{0\}$ & $\{0\}$ & $\{0\}$ & $\mathbb{Z} \backslash N_{0}$ \\
$\{2\}$ & $\{0\}$ & $\{0\}$ & $\varnothing$ & $\{0\}$ & $\mathbb{Z} \backslash N_{0}$ \\
$\{2\}$ & $\{0\}$ & $\{0\}$ & $\varnothing$ & $\{0\}$ & $\mathbb{Z} \backslash N_{0}^{\prime}$
\end{tabular}

Definition 2.5 thus yields $Z(\mathcal{C}) \in Q$ if $\mathcal{C}$ is case $\mathcal{O}$. Hence, the overall claim is true.

Author Contributions This work was part of the first author's Master's thesis supervised by the second author.

Funding Open Access funding enabled and organized by Projekt DEAL. The first author was supported by an IRTG scholarship of the SFB-TRR 195. The second author was supported by the SFB-TRR 195, and by the DFG project Quantenautomorphismen von Graphen.

Availability of data and material Data sharing not applicable to this article as no datasets were generated or analysed during the current study.

\section{Declarations}

Conflict of interests The authors declare that they have no conflict of interest.

Open Access This article is licensed under a Creative Commons Attribution 4.0 International License, which permits use, sharing, adaptation, distribution and reproduction in any medium or format, as long as you give appropriate credit to the original author(s) and the source, provide a link to the Creative Commons licence, and indicate if changes were made. The images or other third party material in this article are included in the article's Creative Commons licence, unless indicated otherwise in a credit line to the material. If material is not included in the article's Creative Commons licence and your intended use is not permitted by statutory regulation or exceeds the permitted use, you will need to obtain permission directly from the copyright holder. To view a copy of this licence, visit http://creativecommons.org/licenses/by/4.0/.

\section{References}

1. Banica, T., Speicher, R.: Liberation of orthogonal Lie groups. Adv. Math. 222, 1461-1501 (2009)

2. Gromada, D.: Classification of globally colorized categories of partitions. Infin. Dimens. Anal. Quantum Probab. Relat. Topic. 21(04), 1850029 (2018)

3. Mang, A., Weber, M.: Categories of two-colored pair partitions part I: categories indexed by cyclic groups. Ramanujan J. 1-28 (2019)

4. Mang, A., Weber, M.: Categories of two-colored pair partitions part II: categories indexed by semigroups. J. Combin. Theory Ser. A 180 (2021) 
5. Mang, A., Weber, M.: Non-hyperoctahedral categories of two-colored partitions part I: new categories. J. Algeb. Combin. 1-39 (2021)

6. Tarrago, P., Weber, M.: The classification of tensor categories of two-colored noncrossing partitions. J. Combin. Theory Ser. A 154, 464-506 (2017)

7. Woronowicz, S.: Compact matrix pseudogroups. Commun. Math. Phys. 111, 613-665 (1987)

8. Woronowicz, S.: Tannaka-Krein duality for compact matrix pseudogroups. Twisted $S U(N)$ groups. Invent. Math. 93, 35-76 (1987)

9. Woronowicz, S.: Compact quantum groups. In: Connes, A. et al. (eds.) Quantum Symmetries/ Symétries Quantiques. Proceedings of the Les Houches Summer School, Session LXIV, Les Houches, France, August 1st-September 8th, 1995, pp. 845-884 (1998)

Publisher's Note Springer Nature remains neutral with regard to jurisdictional claims in published maps and institutional affiliations. 